\title{
Ultrasonic study of molecular interaction in Binary liquid mixture at $308 \mathrm{~K}$
}

\author{
${ }^{1}$ D. Ubagaramary, ${ }^{2}$ Dr.P.Neeraja \\ ${ }^{I}$ Department of Chemistry, Sri venkateswara institute of engineering, krishnagiri, India \\ ${ }^{2}$ Department of Chemistry, Adhiyamann college of engineering, Hosur-635 109, India
}

\begin{abstract}
The ultrasonic velocity $(u)$, density $(\rho)$ and viscosity $(\eta)$ have been measured in binary liquid mixtures containing IBMK+Aniline, IBMK+N,N-Dimethyl aniline,Acetophenone $+C y c l o h e x a n e$ at $308 \mathrm{~K}$. From these data some of acoustical parameters such as adiabaticcompressibility $\left(\beta_{\text {add }}\right)$, free length $(L f)$, free volume $(V f)$ and internal pressure $\left(\pi_{i}\right)$ have been computed using thestandard relations. The excess values of these parameters are also evaluated over the whole concentration range.The result is interpreted in terms of molecular interaction such as dipole-dipole interaction through hydrogen bonding between components of mixtures. The dependence of excess properties of mixture compositions were compared and discuss in terms of the intermolecular free length and other factors affecting the salvation and self association effect. The excess values of these indicate dipole-induced dipole interaction complexity in the binaryliquid mixture.
\end{abstract}

Keywords: molecular interaction, Ultrasonic velocity, adiabatic compressibility, inter-mole molecular free length internal pressure \& dipole - dipole interactions, dipole -induced dipole interactions

\section{Introduction}

In recent years, ultrasonic technique has become a powerful tool for studying the molecular behaviour of liquid mixtures ${ }^{1-3}$. This is because of its ability of characterizing physico-chemical behaviour of liquid medium $^{4-6}$.The measurement of ultrasonic velocity have been adequately employed in understanding the molecular interactions in liquid mixtures. Molecular interaction studies can be carried out by both spectroscopic $^{7,8}$ and non-spectroscopic ${ }^{9,-10}$ techniques. However, ultrasonic velocity ${ }^{11}$ and viscosity ${ }^{12}$ measurements have been widely used in the field of interactions and structural aspect evaluation studies.

The following three binary systems are studied at $308 \mathrm{~K}$.

System-1 IBMK+Aniline

System-2 IBMK+N,N-Dimethyl aniline

System-3 Acetophenone+Cyclohexane

Aspects in Theoretical

\section{Experimental techniques}

1. Adiabatic compressibility ( $\square$ )

The adiabatic compressibility is the fractional decrease of volume per unit increase of pressure, when no heat flows in or out. These changes are related to the compressibility of the medium by thermodynamic relation

$\beta=\frac{1}{v}[\partial v / \partial p]$

It can also be calculated from the speed of sound $(\mathrm{U})$ and the density of the medium $(\rho)$ using the equation of Newton Laplace as

$\beta=\frac{1}{u^{2} \rho}$

\section{Intermolecular free length}

The adiabatic compressibility of a liquid can be expressed in terms of the intermolecular free length which is the distance between the surfaces of the neighboring molecules and is given by the relation,

$\mathbf{L}_{\mathrm{f}}=\mathrm{KT} \square^{1 / 2}$

Where $\mathrm{K}_{\mathrm{T}}$ is the temperature dependent constant. 


\section{Free Volume $\left(\mathbf{V}_{\mathrm{f}}\right)$}

Free volume is one of the significant factors in explaining the variations in the physio-chemical properties of liquids and liquid mixtures. The free space and its dependent properties have close connection with molecular structure and it may show interesting features about interactions, which may occur when two or more liquids are mixed together. This molecular interactions between like and unlike molecules are influenced by structural arrangements along with shape and size of the molecules. A liquid may be treated as if it were composed of individual molecules each moving in a volume $\mathrm{V}_{\mathrm{f}}$ in an average potential due to its neighbors. That is, the molecules of a liquid are not quite closely packed and there are some free spaces between the molecules for movement and the volume $V_{\mathrm{f}}$ is called the free volume ${ }^{13}$.Eyring and Kincaid ${ }^{14}$ defined the free volume as the effective volume in which particular molecule of the liquid can move and obey perfect gas lawsFree volume in terms of Ultrasonic velocity (U) and the Viscosity of the liquid $(\eta)$ as

$\mathbf{V}_{\mathrm{f}}=\left[\frac{M_{e f f} U}{K \eta}\right]^{3 / 2}$

(4)

Where $\mathrm{M}_{\text {eff }}$ is the effective molecular weight

\section{$\mathbf{M}_{\text {eff }}=\sum \mathbf{X i M i}$}

in which $\mathrm{m}_{\mathrm{i}}$ and $\mathrm{X}_{\mathrm{i}}$ are

the molecular weight and the mole fraction of the individual constituents respectively). $\mathrm{K}$ is a temperature independent, constant which is equal to $4.28 \times 10^{9}$ for all liquids.

\section{Internal Pressure $(\square)$}

The measurement of internal pressure is important in the study of the thermodynamic properties of liquids. The internal pressure is the cohesive force, which is a resultant of force of attraction and force of repulsion between the molecules ${ }^{15,16}$. Cohesion creates a pressure within the liquid of value between 103 and 104 atmosphere. Internal pressure also gives an idea of the solubility characteristics. Dissolved solutes exist under the internal pressure of the medium and their interactions with the solvent arise through hydrogen bonding, charge transfer, Columbic (or) Vanderwaal's interaction. The term $\mathrm{a} / \mathrm{v}^{2}$ in Vanderwaal's ${ }^{17}$ equation being the measure of attractive force of the molecule is called the cohesive (or) internal pressure.

The internal pressure is the single factor which varies due to all type of solvent-solute, solute-solute and solventsolvent interactions. A general method of measuring the internal pressure based on the Maxwell's equation of thermodynamics ${ }^{18}$ is

$$
\mathbf{P}=\mathbf{T}\left[\frac{\partial P}{\partial T}\right]_{V}-\left[\frac{\partial E}{\partial V}\right]_{T}
$$

On the basis of statistical thermodynamics, expression for the determination of internal pressure by the use of free volume concept as given by

$$
\mathbf{V}_{\mathrm{f}}=\frac{1}{V^{2}}\left[\frac{b R T}{P+\left(\frac{\partial E}{\partial v}\right)_{T}}\right]^{3}
$$

As $\left(\frac{\partial E}{\partial v}\right)_{T}$ is the internal pressure and neglecting $\mathrm{P}$ which is insignificantly small to $\pi_{\mathrm{i}}$

$$
\mathrm{V}_{\mathrm{f}}=\frac{1}{V^{2}}\left[\frac{b R T}{\pi_{i}}\right]^{3}
$$

The final equation for the evaluation of internal pressure can be obtained by combining and rearranging the equations (6) and (7)

$$
\square_{\mathrm{i}=\mathrm{bRT}}\left(\frac{K \eta}{U}\right)^{\frac{1}{2}}\left(\frac{\rho^{\frac{2}{3}}}{M_{e f f^{\frac{7}{6}}}}\right)
$$

Where $\mathrm{K}$ is a constant, $\mathrm{T}$ the absolute temperature, $\eta$, the viscosity in $\mathrm{NSm}^{-2}, \mathrm{U}$, the ultrasonic velocity in $\mathrm{ms}^{-1}, \rho$, the density in $\mathrm{kgm}-3$ of the liquid. 


\section{Relaxation time ( $\square$}

Relaxation time is the time taken for the excitation energy to appear as translational energy and it depends on temperature and on impurities.

The dispersion of the ultrasonic velocity in binary mixture reveals information about the Characteristic time of the relaxation process that causes dispersion. The relaxation time $(\tau)$ can be calculated from the relation.

$\square=\frac{4}{3}$

\section{Acoustic Impedance (Z)}

The Specific acoustic impedance is given by

$\mathbf{Z}=\mathbf{U}^{*}$

Where $\mathrm{U}$ and $\rho$ are velocity and density of liquid respectively.

\section{Gibb's Free Energy ( $\left.\square G^{*}\right)$}

The relaxation time for a given transition is related to the activation free energy. The variation of $\square$ with temperature can be expressed in the form of Eyring salt process theory ${ }^{19}$.

$\frac{1}{\tau}=\frac{K T}{h} \exp \left(\frac{-\Delta G^{*}}{K T}\right)$

The above equation can be rearranged as,

$\Delta G^{*}=K T \log \left(\frac{h}{K T \tau}\right)$

Where $\mathrm{K}$ is the Boltzmann constant and $\mathrm{h}$ is plank's constant.

\section{Excess parameters}

The excess values are calculated using the formula,

$A_{E X C E S S}=A_{E X P}-A_{I D E A L}$

Where, $\mathbf{A}_{\mathbf{i d}}=\sum \mathbf{A}_{\mathbf{i}} \mathbf{X}_{\mathbf{i}}$, where $\mathrm{A}_{\mathrm{i}}$ is any acoustical parameter and $\mathrm{X}_{\mathrm{i}}$ is the mole fraction of liquid component.

\section{Density Measurement}

\section{Experimental}

The density of pure liquids and mixtures are measured using a $10 \mathrm{ml}$ specific gravity bottle.

The specific gravity bottle with the experimental liquid is immersed in a temperature controlled water bath. The densities of pure liquids thus obtained are found to be in good agreement with standard values. The measured density was measured using the formula,

$\rho_{2}=\frac{w_{2}}{w_{1}} \rho_{1}$

Where,

$\mathbf{W}_{\mathbf{1}}$, is the weight of the distilled water.

$\mathbf{W}_{2}$ is the weight of the experimental liquid

$\square_{\mathbf{1}}$, is the density of water.

$\square_{2}$ is the experimental liquid.

\section{Viscosity measurement}

The viscosity of the pure liquids and liquid mixtures are measured using an Ostwald's Viscometer calibrated with doubly distilled water. The Ostwald's Viscometer with the experimental liquid is immersed in a temperature controlled water bath. The time of flow was measured using a Racer stop watch with an accuracy of $0.1 \mathrm{sec}$. Viscosity was determined using the relation

$\eta_{2}=\boldsymbol{\eta}_{1}\left(\frac{t_{2}}{t_{1}}\right)\left(\frac{\rho_{2}}{\rho_{1}}\right)$

Where,

$\boldsymbol{\eta}_{\mathbf{2}}$ is the Viscosity of water

$\boldsymbol{t}_{\mathbf{1}}$, is the time of flow of water 
$\rho_{1}$ is the density of water.

$\boldsymbol{\eta}_{1}$ is the viscosity of the experimental liquid.

$\boldsymbol{t}_{2}$ is the time of flow of the experimental liquid.

$\boldsymbol{\rho}_{2}$ is the density of the experimental liquid.

\section{Velocity Measurement}

The velocity of ultrasonic waves in the liquid mixture have been measured using an ultrasonic interferometer (Mittal Enterprises, New Delhi) working at a fixed frequency of $2 \mathrm{MHZ}$ with a tolerance of \pm $0.005 \%$. The measuring cell is a specially designed double walled vessel with provision for temperature constancy. The high frequency generator excitesa quartz crystal fixed at the bottom of the measuring cell, at its resonant frequency. The capacity of the measuring cell is $12 \mathrm{cc}$. A fine micrometer screw, with a least count of $0.01 \mathrm{mmat}$ the top of the cell, can be raised (or) lowered the reflector plate in the liquid through a known distance. The measuring cell is connected to the output terminals of the high frequency generator through a shielded cable. Ultrasonic waves, normal to quartz crystal, is reflected from the reflector plate. Stationary waves are formed in the region between reflector plate and the quartz crystal. The micrometer is slowly moved till a number of maximum readings ( $\mathrm{n}$ ) of the anode current is passed. The total distance moved by the micrometer is noted (d). The wavelength of the ultrasonic waves in the liquid is $\lambda=2 \mathrm{~d} / \mathrm{n}$. The velocity of ultrasonicwaves in the liquid $\mathrm{U}=\lambda \mathrm{f}$. Where $\mathrm{f}$ is the frequency of the generator.

\section{Results and Discussion}

The experimental values of density viscosity, ultrasonic velocity for the three binary systems IBMK+Aniline, IBMK+N,N-Dimethyl aniline Acetophenone+Cyclohexane at 308K are given in the tables 1, $2 \& 3$.The parameters adiabatic compressibility $\left(\beta_{\mathrm{ad}}\right)$, free length $\mathrm{L}_{\mathrm{f}}$, free volume $\left(\mathrm{V}_{\mathrm{f}}\right)$, acousticimpedance $(\mathrm{Z})$, internal pressure $\left(\pi_{\mathrm{i})}\right.$, relaxation time $(\tau)$ at $308 \mathrm{~K}$ are listed in tables $4,5,6,7,8 \& 9$.

\section{SYSTEM:-1 IBMK -Aniline}

Table:-1 Mole fraction of first component $\left(X_{1}\right)$, Mole fraction of second component $\left(X_{2}\right)$, Density $(\rho)$, viscosity $(\eta)$, ultrasonic velocity(U), acoustic impedance(Z), Leonard's Jones potential(LJP) and Molecular interaction parameter $\left(\chi_{\mathrm{u}}\right)$ values at different mole fraction of IBMK -Aniline at $308 \mathrm{~K}$.

\begin{tabular}{|c|c|c|c|c|c|c|c|}
\hline \multicolumn{2}{|c|}{ Mole fraction } & \multirow{2}{*}{$\begin{array}{l} \\
(\mathrm{g} / \mathrm{cm} 3)\end{array}$} & \multirow{2}{*}{$\begin{array}{l}\square \\
\text { (cp) }\end{array}$} & \multirow{2}{*}{$\begin{array}{l}\mathbf{U} \\
(\mathrm{m} / \mathrm{s})\end{array}$} & \multirow{2}{*}{$\begin{array}{l}\mathrm{Z} \\
\left(\mathrm{gm}^{-2} \mathrm{~s}^{-1}\right)\end{array}$} & \multirow{2}{*}{ LJP } & \multirow{2}{*}{$\begin{array}{l}\square u \\
(\mathbf{m} / \mathbf{s})\end{array}$} \\
\hline $\mathbf{X}_{1}$ & $\mathbf{X}_{2}$ & & & & & & \\
\hline 0.0000 & 1.0000 & 1.0141 & 1.5777 & 1380.00 & 1399.4614 & 43.6364 & 0.0007 \\
\hline 0.0509 & 0.9491 & 1.0016 & 1.4937 & 1373.00 & 1375.2025 & 42.2907 & 0.0007 \\
\hline 0.1020 & 0.8980 & 0.9872 & 1.4368 & 1364.00 & 1346.5473 & 40.6780 & 0.0007 \\
\hline 0.1541 & 0.8459 & 0.9731 & 1.3814 & 1352.00 & 1315.6294 & 38.7097 & 0.0007 \\
\hline 0.2738 & 0.7262 & 0.9421 & 1.2353 & 1324.00 & 1247.3346 & 34.7826 & 0.0007 \\
\hline 0.3347 & 0.6653 & 0.9273 & 1.1620 & 1305.00 & 1210.1290 & 32.5424 & 0.0007 \\
\hline 0.4560 & 0.5440 & 0.8991 & 1.0346 & 1275.00 & 1146.3522 & 29.5385 & 0.0008 \\
\hline 0.5229 & 0.4771 & 0.8842 & 0.9783 & 1262.00 & 1115.8667 & 28.4024 & 0.0008 \\
\hline 0.6732 & 0.3268 & 0.8535 & 0.9001 & 1241.00 & 1059.2000 & 26.7409 & 0.0008 \\
\hline $\mathbf{0 . 8 2 2 5}$ & 0.1775 & 0.8256 & 0.7966 & 1226.00 & 1012.1812 & 25.6684 & 0.0008 \\
\hline 0.9179 & 0.0821 & 0.8156 & 0.4523 & 1210.00 & 986.8708 & 24.6154 & 0.0008 \\
\hline 1.0000 & 0.0000 & 0.7940 & 0.3947 & 1204.00 & 955.9720 & 24.2424 & 0.0008 \\
\hline
\end{tabular}


SYSTEM:-1 IBMK+ Aniline

Fig. 1 Molefraction vs U

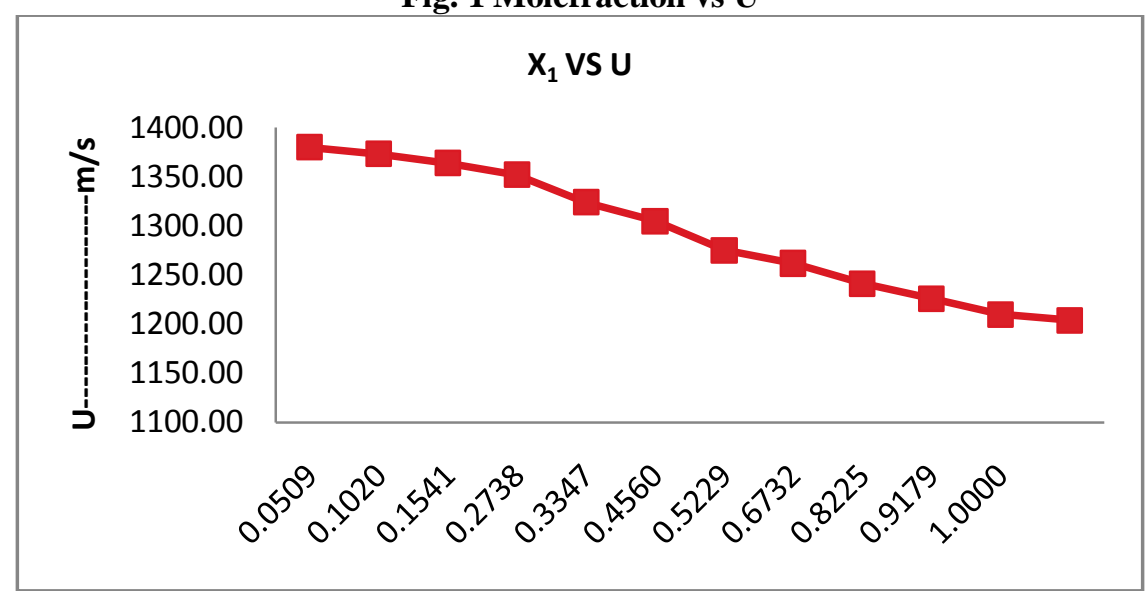

\section{MOLE FRACTION OF FIRST COMPONENT}

Fig.2 Molefraction vs $\square_{\text {u }}$

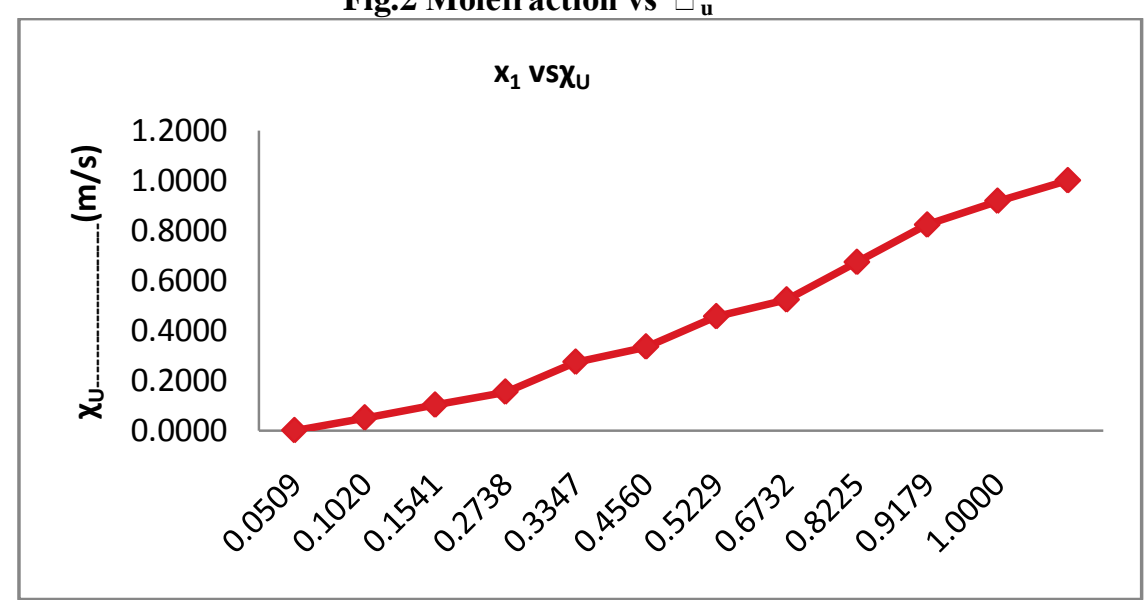

\section{MOLE FRACTION OF FIRST COMPONENT}

Table:-2 adiabatic compressibility $(\beta)$, relaxation time $(\tau)$, free volume $\left(\mathrm{V}_{\mathrm{f}}\right)$, internal pressure $\left(\pi_{\mathrm{i}}\right)$, cohessive force $(C E)$, absorption co-efficient $\left(\alpha / \mathrm{f}^{2}\right)$, free length $\left(\mathrm{L}_{\mathrm{f}}\right) \&$ activation energy $\left(\Delta \mathrm{G}^{\#}\right)$ values at different mole fraction of IBMK+Aniline at $308 \mathrm{~K}$.

\begin{tabular}{|c|c|c|c|c|c|c|c|}
\hline $\begin{array}{l}\square \mathbf{1 0}^{-10} \\
\left(\mathrm{~T} . \mathrm{Pa}^{)^{-1}}\right.\end{array}$ & $\begin{array}{l}\square 0^{6} \\
\text { (s) }\end{array}$ & $\begin{array}{l}V_{\mathrm{f}} \\
(\mathrm{ml} / \mathrm{mole})\end{array}$ & $\begin{array}{l}\square_{\mathrm{i}} \\
\text { (atm) }\end{array}$ & $\begin{array}{l}\text { CE } \\
\text { (gJ/mole) }\end{array}$ & $\begin{array}{l}\square / \mathbf{f}^{\mathbf{1} 10}{ }^{3} \\
\left(\mathbf{N P m}^{-1} \mathbf{s}^{2}\right)\end{array}$ & $\begin{array}{l}\mathbf{L}_{\mathrm{f}} \\
\left(\mathrm{T} . \mathrm{Pa}^{)-1}\right.\end{array}$ & $\begin{array}{l}\square \mathbf{G}^{\#} 10^{-20} \\
(\mathrm{gj} / \text { mole })\end{array}$ \\
\hline 51.78 & 1.0892 & 0.0830 & 5695.1056 & 523.0095 & 51.2009 & 114.4098 & 2.9106 \\
\hline 52.96 & 1.0548 & 0.0900 & $\mathbf{5 4 8 5 . 2 2 9 1}$ & 511.9781 & 46.2289 & 115.4145 & 2.9046 \\
\hline 54.45 & 1.0430 & 0.0950 & 5321.7723 & 505.9004 & 43.3139 & 116.6361 & 2.9026 \\
\hline 56.22 & 1.0355 & 0.1000 & 5167.7200 & 500.3194 & 40.9071 & 117.9986 & 2.9012 \\
\hline 60.55 & 0.9973 & 0.1161 & 4782.9528 & 482.5727 & $\mathbf{3 4 . 4 6 4 7}$ & 121.1860 & 2.8943 \\
\hline 63.32 & 0.9811 & 0.1254 & 4599.3381 & 473.5706 & 31.8063 & 123.0348 & 2.8913 \\
\hline 68.42 & 0.9438 & 0.1461 & 4256.8445 & 456.0857 & 26.8274 & 126.4110 & 2.8841 \\
\hline 71.01 & 0.9263 & 0.1576 & 4091.3081 & 447.9071 & 24.5716 & 128.1261 & 2.8807 \\
\hline 76.08 & 0.9130 & 0.1770 & 3816.7294 & 437.5947 & 21.4349 & 131.5088 & 2.8780 \\
\hline 80.58 & 0.8559 & 0.2121 & 3489.6613 & 418.0509 & 17.0034 & 134.5286 & 2.8661 \\
\hline 83.74 & 0.5050 & 0.4910 & 2604.8404 & 318.0172 & 5.6640 & 136.2428 & 2.7686 \\
\hline 86.88 & 0.4572 & 0.6030 & 2380.0482 & 300.2051 & 4.3024 & 138.4271 & 2.7503 \\
\hline
\end{tabular}


Fig.3 Molefraction vs

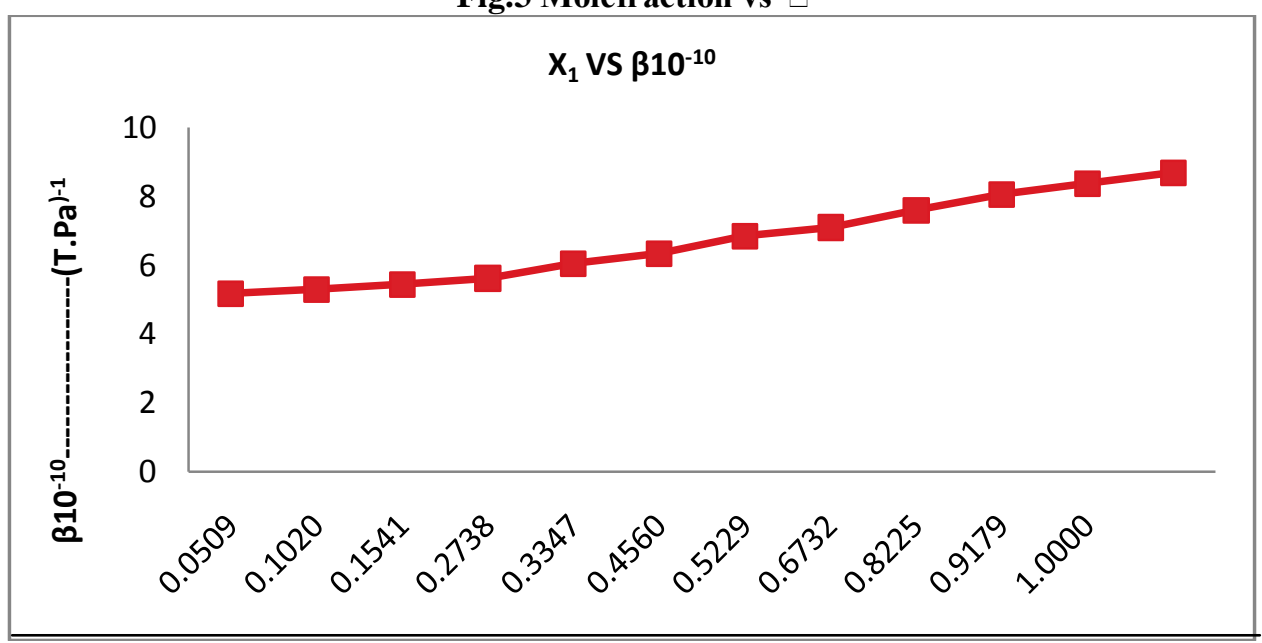

\section{MOLE FRACTION OF FIRST COMPONENT}

Fig.4 Molefraction vs

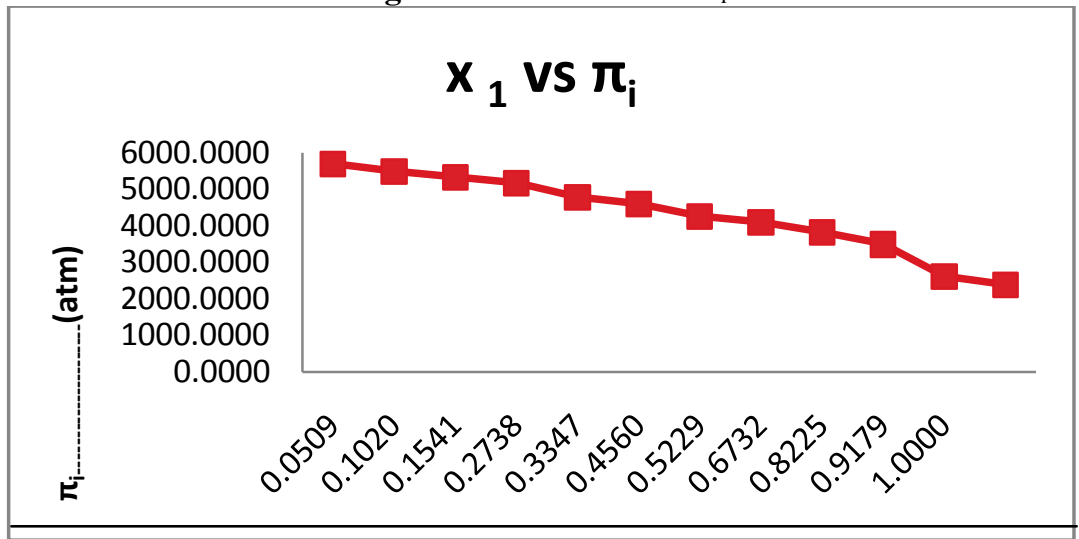

MOLE FRACTION OF FIRST COMPONENT

Fig.5 Molefraction vs CE

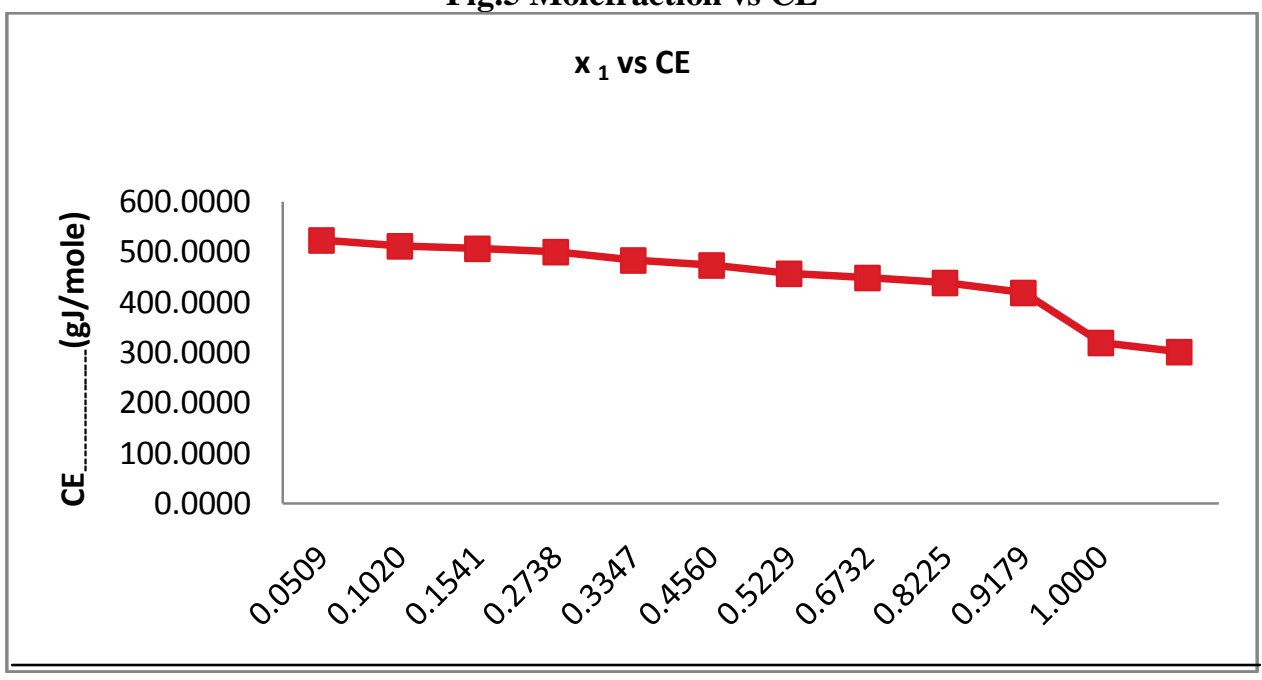

MOLE FRACTION OF FIRST COMPONENT 
Fig.6 Mole fraction vs $\Delta G^{\neq}$

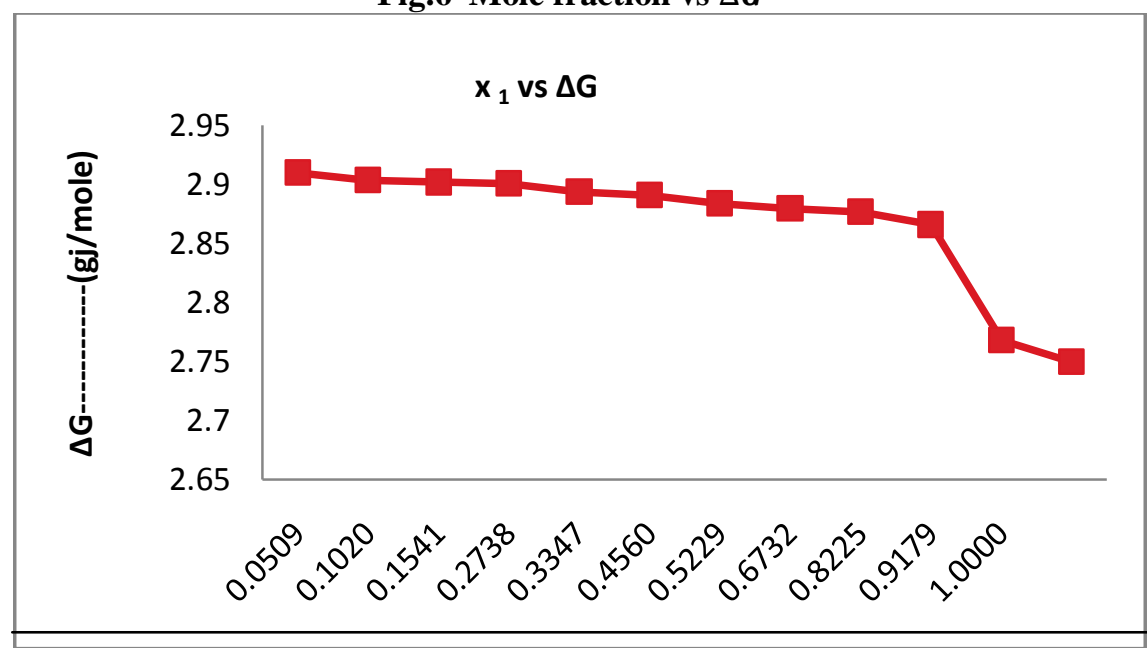

MOLE FRACTION OF FIRST COMPONENT

Table:-3 Excess ultrasonic velosity $\left(\mathrm{U}^{\mathrm{E}}\right)$, Excess viscocity $\left(\eta^{\mathrm{E}}\right)$, Excess available volume $\left(\mathrm{VA}^{\mathrm{E}}\right)$,Excess acoustical impedance $\left(\mathrm{Z}^{\mathrm{E}}\right)$, Excess volume $\left(\mathrm{V}^{\mathrm{E}}\right)$,Excess adiabatic compressibility $\left(\beta^{\mathrm{E}}\right)$,Excess free length

$\left(\mathrm{L}_{\mathrm{F}}^{\mathrm{E}}\right)$ and Excess free volume $\left(\mathrm{V}_{\mathrm{F}}^{\mathrm{E}}\right)$ values at various mole fractions of IBMK + aniline at 308K.

\begin{tabular}{|c|c|c|c|c|c|c|c|}
\hline $\begin{array}{l}\mathbf{U}^{\mathrm{E}} \\
(\mathbf{m} / \mathbf{s})\end{array}$ & $\begin{array}{l}\mathrm{E} \\
\left(\mathrm{NS} / \mathrm{cm}^{2}\right)\end{array}$ & $\begin{array}{l}\mathrm{VA}^{\mathrm{E}} \\
\left(\mathrm{cm}^{3}\right)\end{array}$ & $\begin{array}{l}Z^{E} \\
\left(g^{-2} s^{-1}\right)\end{array}$ & $\begin{array}{l}V^{\mathrm{E}} \\
\left(\mathrm{cm}^{3} / \mathrm{mole}\right)\end{array}$ & $\begin{array}{c}{ }^{\mathrm{E}} \mathbf{1 0}^{-9} \\
\left(\mathrm{~g}-{ }^{-1} \mathrm{~ms}^{2}\right) \\
\end{array}$ & $\begin{array}{l}V_{\mathbf{F}}^{\mathrm{E}} \\
\left(\mathrm{cm}^{3}\right)\end{array}$ & $\begin{array}{l}\mathbf{L}_{\mathbf{F}}^{E} \\
(\mathbf{c m})\end{array}$ \\
\hline 0.0 & 0.0000 & 0.0000 & 0.0000 & 0.0000 & 0.00 & 0.0000 & 0.0000 \\
\hline 2.0 & $\begin{array}{l}-0.0238 \\
\end{array}$ & \begin{tabular}{|c|}
-0.3313 \\
\end{tabular} & -1.6853 & -0.2433 & -6.04 & -0.0195 & -0.2178 \\
\hline 2.0 & -0.0202 & $\begin{array}{l}-0.5019 \\
\end{array}$ & -7.6781 & -0.2712 & -9.14 & -0.0411 & -0.2235 \\
\hline-0.9 & -0.0140 & -0.4856 & -15.4902 & -0.3042 & -9.68 & -0.0631 & -0.1122 \\
\hline-7.8 & -0.0185 & -0.3132 & -30.6994 & -0.3317 & -8.38 & -0.1092 & 0.2002 \\
\hline-16.1 & -0.0197 & 0.1345 & -40.8965 & -0.3498 & -2.05 & -0.1316 & 0.5864 \\
\hline-24.7 & -0.0037 & 0.6584 & -50.8780 & -0.3334 & 6.33 & $\begin{array}{l}-0.1740 \\
\end{array}$ & 1.0493 \\
\hline-26.0 & 0.0192 & 0.7787 & -51.6941 & -0.2920 & 8.78 & -0.1973 & 1.1577 \\
\hline-20.5 & 0.1188 & 0.5823 & -41.7043 & -0.2730 & 6.67 & -0.2561 & 0.9305 \\
\hline-9.2 & 0.1919 & 0.0842 & -22.5102 & -0.2486 & -6.51 & -0.2986 & 0.3646 \\
\hline-8.4 & -0.0395 & 0.0668 & -5.5117 & -1.2306 & -2.54 & -0.0693 & -0.2125 \\
\hline 0.0000 & 0.0000 & 0.0000 & 0.0000 & 0.0000 & 0.0000 & 0.0000 & 0.0000 \\
\hline
\end{tabular}

Fig.7 Molefraction vs $\mathbf{V}^{\mathrm{E}}$

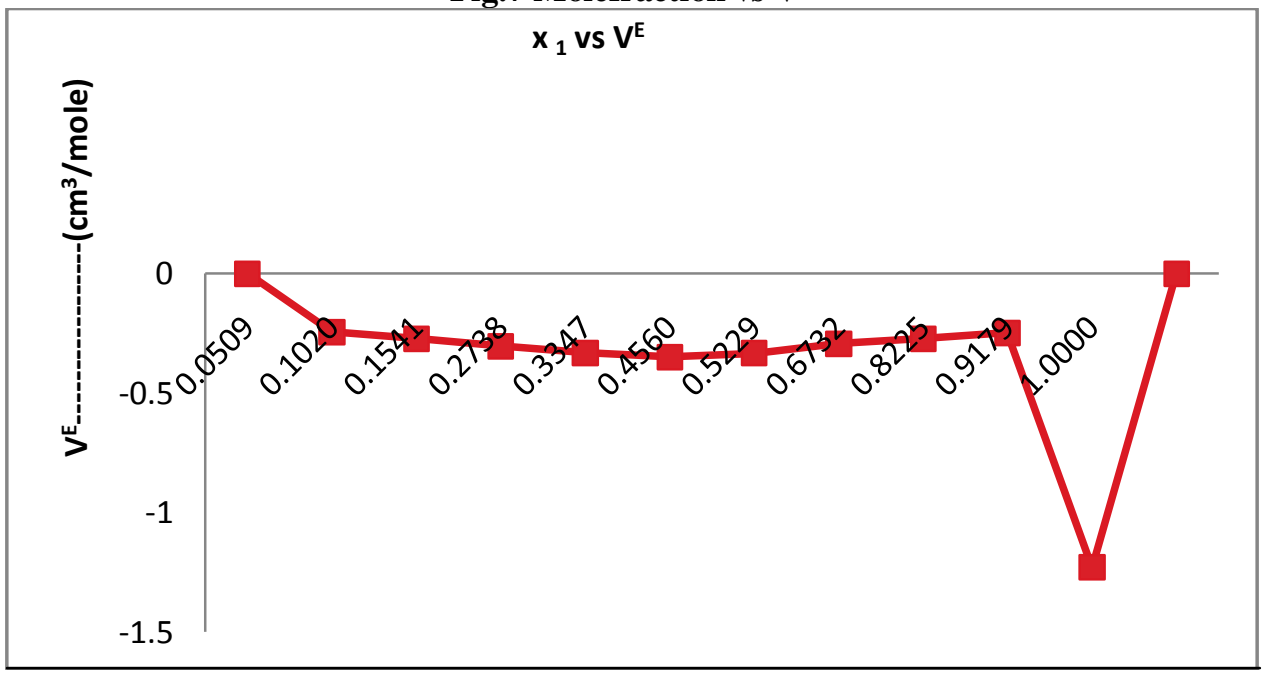

MOLE FRACTION OF FIRST COMPONENT 
Fig.8 Mole fraction vs $Z^{\mathrm{E}}$

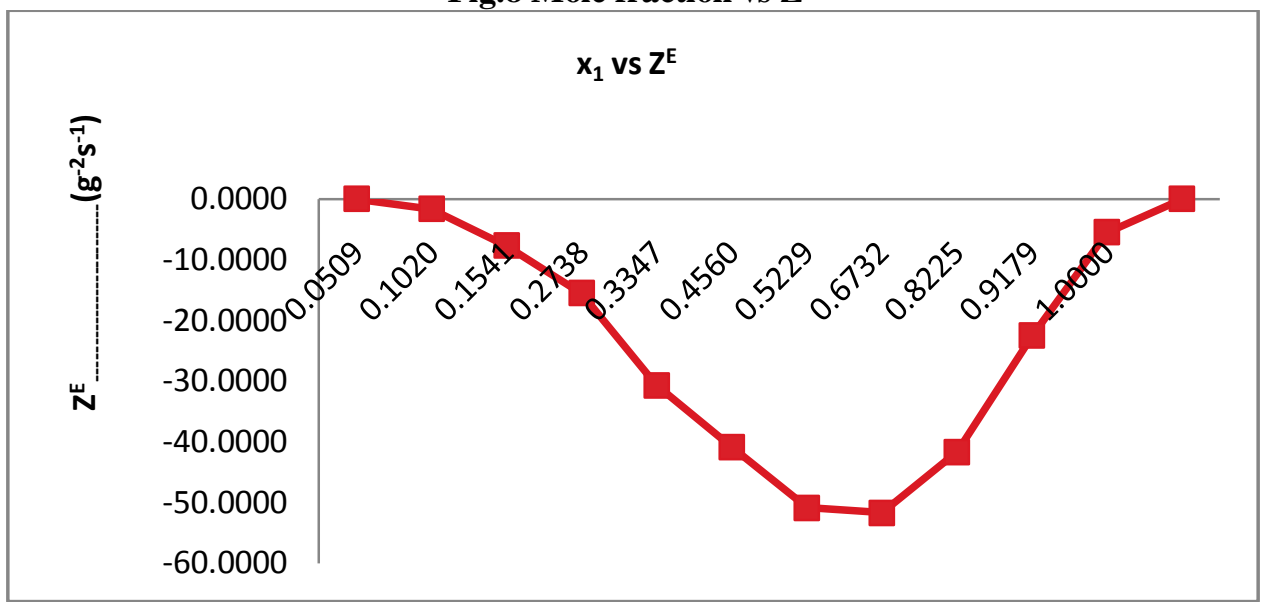

MOLE FRACTION OF FIRST COMPONENT

Fig.9 Mole fraction vs $V_{F}^{E}$

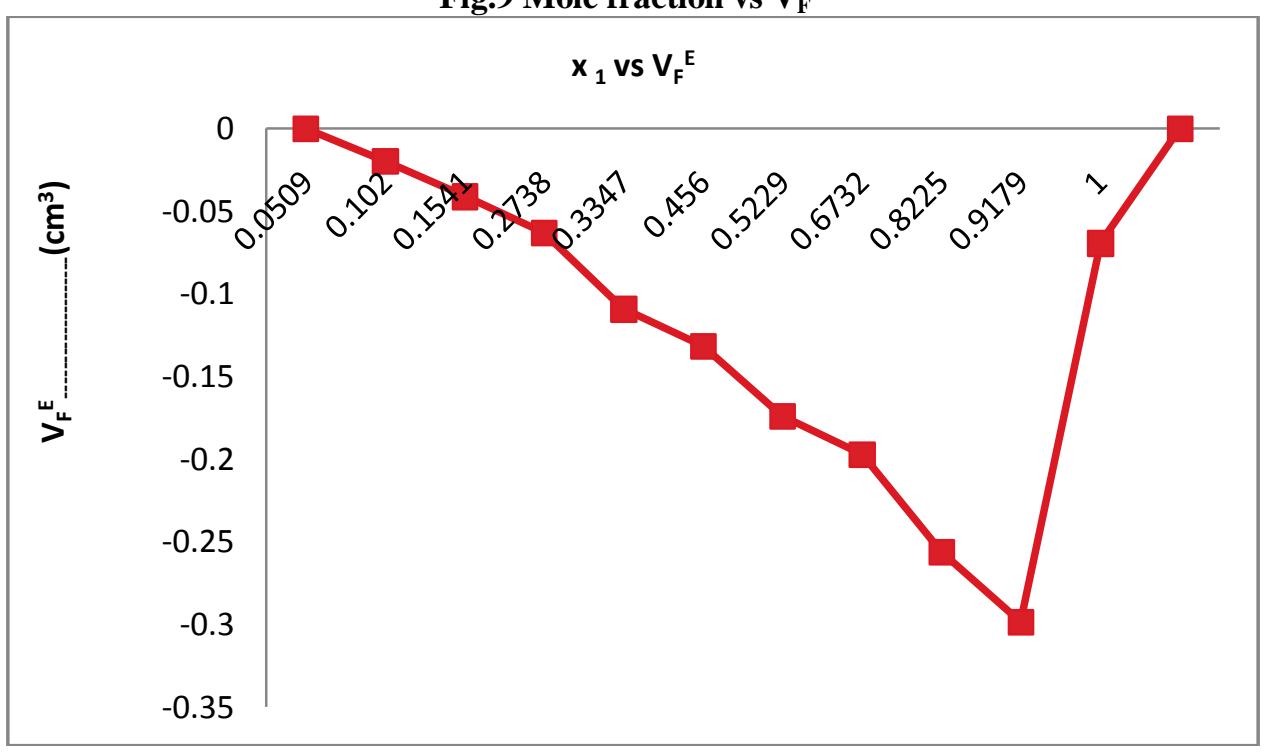

MOLE FRACTION OF FIRST COMPONENT

Fig.10 Mole fraction vs $L_{F}$

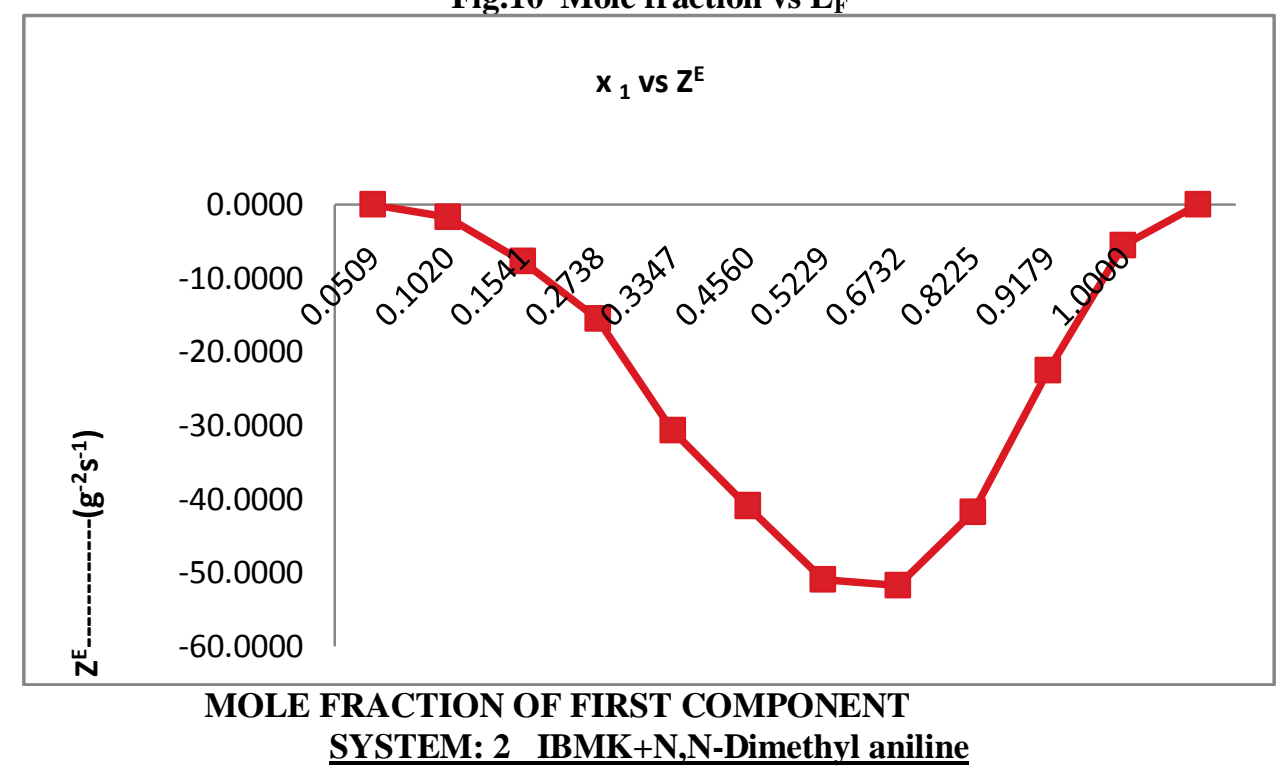


Table:-4 Mole fraction of first component $\left(X_{1}\right), \quad$ Mole fraction of second $\operatorname{component}\left(X_{2}\right)$, Density $(\rho)$, $\operatorname{viscosity}(\eta)$, ultrasonic velocity(U), acoustic impedance(Z), Leonard's Jones potential(LJP) and Molecular interaction parameter $\left(\chi_{u}\right)$ values at different mole fraction of IBMK+_N,N- Dimethyl aniline at 308K.

\begin{tabular}{|c|c|c|c|c|c|c|c|}
\hline \multicolumn{2}{|c|}{ Mole fraction } & \multirow{2}{*}{$\begin{array}{l}\square \\
(\mathrm{g} / \mathrm{cm} 3)\end{array}$} & \multirow{2}{*}{$\begin{array}{l}\square \\
\text { (cp) }\end{array}$} & \multirow{2}{*}{$\begin{array}{l}\mathbf{U} \\
(\mathbf{m} / \mathbf{s})\end{array}$} & \multirow{2}{*}{$\begin{array}{l}Z \\
\left(\mathrm{gm}^{-2} \mathrm{~s}^{-1}\right)\end{array}$} & \multirow{2}{*}{ LJP } & \multirow{2}{*}{$\begin{array}{l}\square \mathrm{u} \\
(\mathbf{m} / \mathbf{s})\end{array}$} \\
\hline$X_{1}$ & $\mathbf{X}_{2}$ & & & & & & \\
\hline 0.0000 & 1.0000 & 0.9408 & 1.4368 & 1393.00 & 1310.5362 & 46.3768 & 0.0000 \\
\hline 0.1366 & 0.8634 & 0.9243 & 1.1515 & 1371.00 & 1267.2135 & 41.9214 & $\mathbf{0 . 0 0 2 8}$ \\
\hline 0.1980 & 0.8020 & 0.9156 & 1.0334 & 1362.00 & 1247.0505 & 40.3361 & $\mathbf{0 . 0 0 4 7}$ \\
\hline 0.3391 & 0.6609 & 0.8951 & 0.9106 & 1337.00 & 1196.7427 & 36.5019 & 0.0061 \\
\hline 0.4057 & 0.5943 & 0.8854 & 0.8206 & 1336.00 & 1182.8920 & 36.3636 & 0.0149 \\
\hline 0.5318 & 0.4682 & 0.8667 & 0.7176 & 1294.00 & 1121.5114 & 31.3725 & 0.0012 \\
\hline 0.6086 & 0.3914 & 0.8552 & 0.6683 & 1271.00 & 1086.9643 & 29.1793 & -0.0055 \\
\hline 0.7421 & 0.2579 & 0.8535 & 0.5935 & 1247.00 & 1064.3210 & 27.1955 & -0.0046 \\
\hline 0.8586 & 0.1414 & 0.8351 & 0.5287 & 1227.00 & 1024.6696 & 25.7373 & -0.0030 \\
\hline 0.9087 & 0.0913 & 0.8176 & 0.4523 & 1210.00 & 989.2934 & 24.6154 & -0.0092 \\
\hline 1.0000 & 0.0000 & 0.7937 & 0.3945 & 1204.00 & 955.6189 & 24.2424 & 0.0000 \\
\hline
\end{tabular}

SYSTEM: 2 IBMK+N,N-Dimethyl aniline

Fig.11 Molefraction vs $\mathrm{U}$

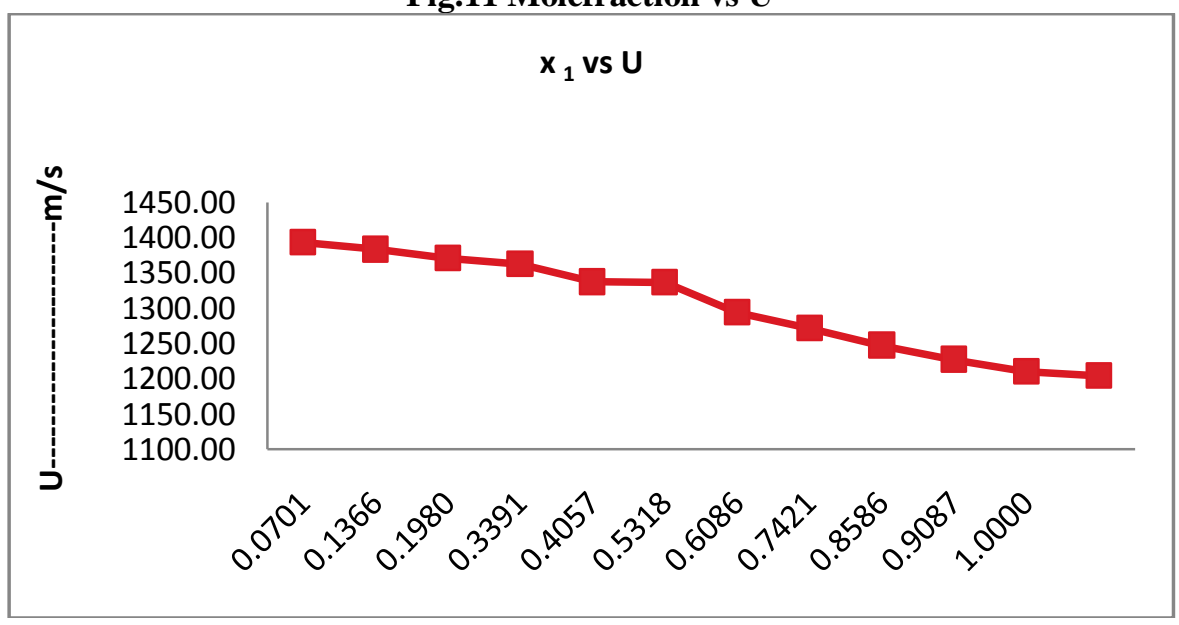

MOLE FRACTION OF FIRST COMPONENT

Fig.12 Mole fraction vs

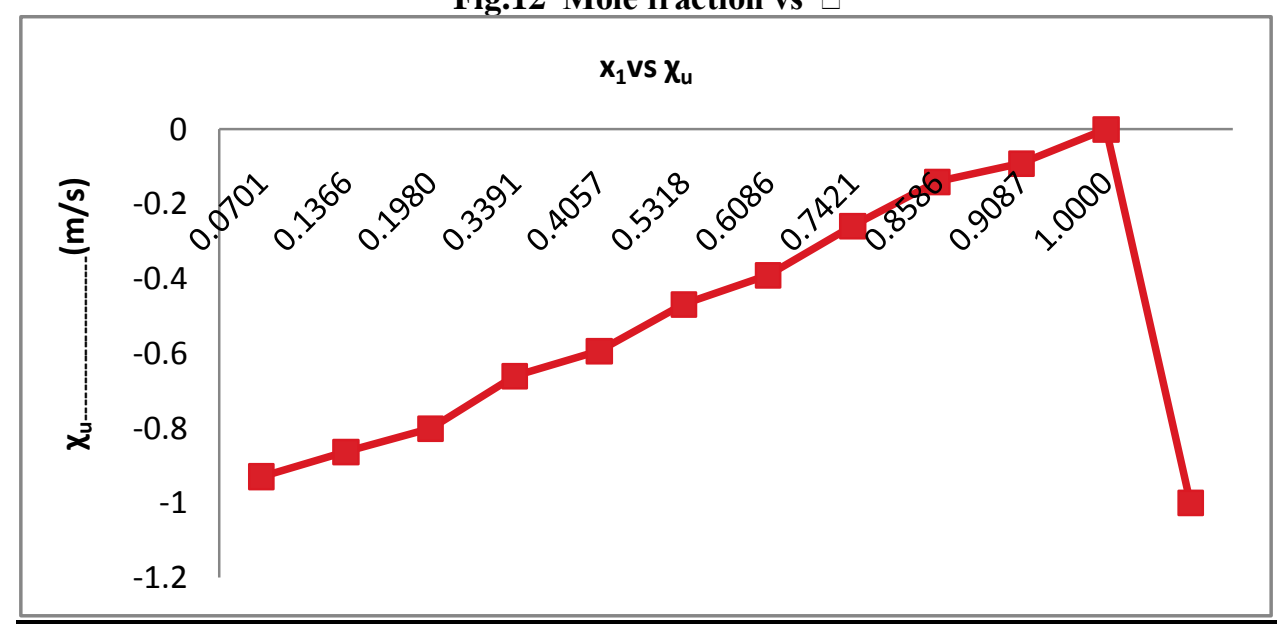

MOLE FRACTION OF FIRST COMPONENT 
Table:-5 Adiabatic compressibility $(\beta)$, Rrelaxation time $(\tau)$, Free volume $\left(\mathrm{V}_{\mathrm{f}}\right)$, Internal pressure $\left(\pi_{\mathrm{i}}\right)$, Cohessive force $(C E)$, Absorption co-efficient $\left(\alpha / f^{2}\right)$, Free length $\left(L_{f}\right) \&$ Activation energy $\left(\Delta G^{\#}\right)$ values at different

\begin{tabular}{|c|c|c|c|c|c|c|c|}
\hline $\begin{array}{c}\square_{10}^{-12} \\
\left(\mathbf{T} . \mathbf{P a}^{\text {)-1 }}\right.\end{array}$ & $\begin{array}{c}\square \mathbf{1 0}^{\mathbf{6}} \\
(\mathrm{s})\end{array}$ & $\begin{array}{c}\mathbf{V}_{\mathbf{f}} \\
(\mathbf{m l} / \mathbf{m o l e})\end{array}$ & $\begin{array}{c}\square_{\mathrm{i}} \\
\text { (atm) }\end{array}$ & $\begin{array}{c}\mathrm{CE} \\
\text { (gJ/mole) }\end{array}$ & $\begin{array}{c}\square / \mathbf{f}^{2} 10^{3} \\
\left(\mathrm{NPm}^{1} \mathbf{s}^{2}\right)\end{array}$ & $\begin{array}{c}\mathbf{L}_{\mathbf{f}} \\
\left(\mathbf{T} . \mathbf{P a}^{)-1}\right.\end{array}$ & $\begin{array}{c}\square \mathbf{G 1 0}^{20} \\
\text { (gj/mole) }\end{array}$ \\
\hline 54.78 & 0.1049 & 0.1438 & 3784.6947 & 487.4880 & 21.5812 & 118.2277 & 2.9037 \\
\hline 55.99 & 9.6949 & 0.1625 & 3644.9520 & 467.2522 & 18.6268 & 119.0981 & 2.8891 \\
\hline 57.56 & 8.8372 & 0.1888 & 3470.9930 & 444.2761 & 15.5316 & 120.2317 & 2.8720 \\
\hline 58.88 & 8.1123 & 0.2163 & 3320.5473 & 424.3735 & 13.1346 & 121.1998 & 2.8562 \\
\hline 62.50 & 7.5881 & 0.2447 & 3193.1398 & 406.8544 & $\mathbf{1 1 . 5 7 3 6}$ & 123.7210 & 2.8438 \\
\hline 63.28 & 6.9234 & 0.2805 & 3054.1431 & 388.5749 & 9.6677 & 124.4432 & 2.8269 \\
\hline 68.91 & 6.5930 & 0.3155 & 2941.6564 & 373.3364 & 8.8179 & 127.8033 & 2.8179 \\
\hline 72.98 & 6.4498 & 0.3342 & 2888.4123 & 366.0523 & 8.4674 & 129.8184 & 2.8138 \\
\hline 75.35 & 5.9624 & 0.3731 & 2829.7340 & 350.0217 & 7.6573 & 131.1920 & 2.7993 \\
\hline 79.54 & 5.6069 & 0.4181 & 2727.3218 & 336.7870 & 6.7979 & 133.7063 & 2.7879 \\
\hline 83.54 & 5.0379 & 0.5096 & 2534.8068 & 316.4488 & 5.3504 & 136.0759 & 2.7682 \\
\hline 86.91 & 4.5717 & 0.6035 & 2378.8592 & 300.1660 & 4.2975 & 138.4527 & 2.7502 \\
\hline
\end{tabular}

Fig.13 Molefraction vs

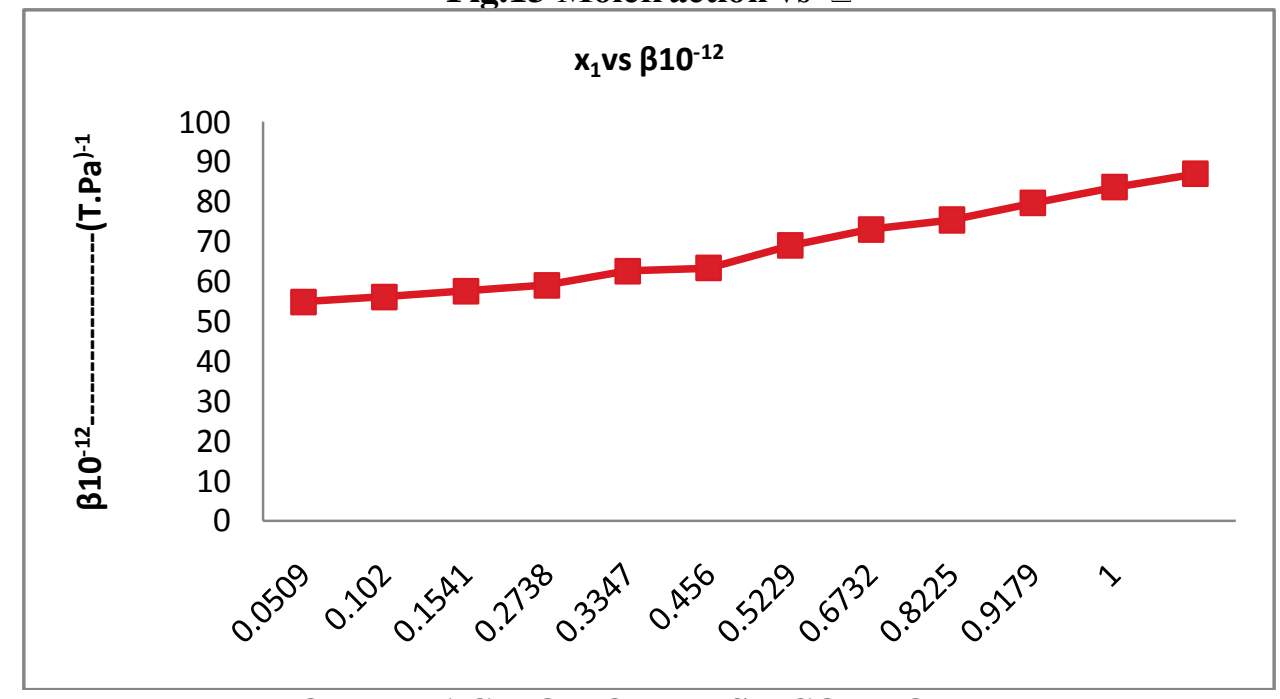

MOLE FRACTION OF FIRST COMPONENT

Fig.14 Mole fraction vs $\square$

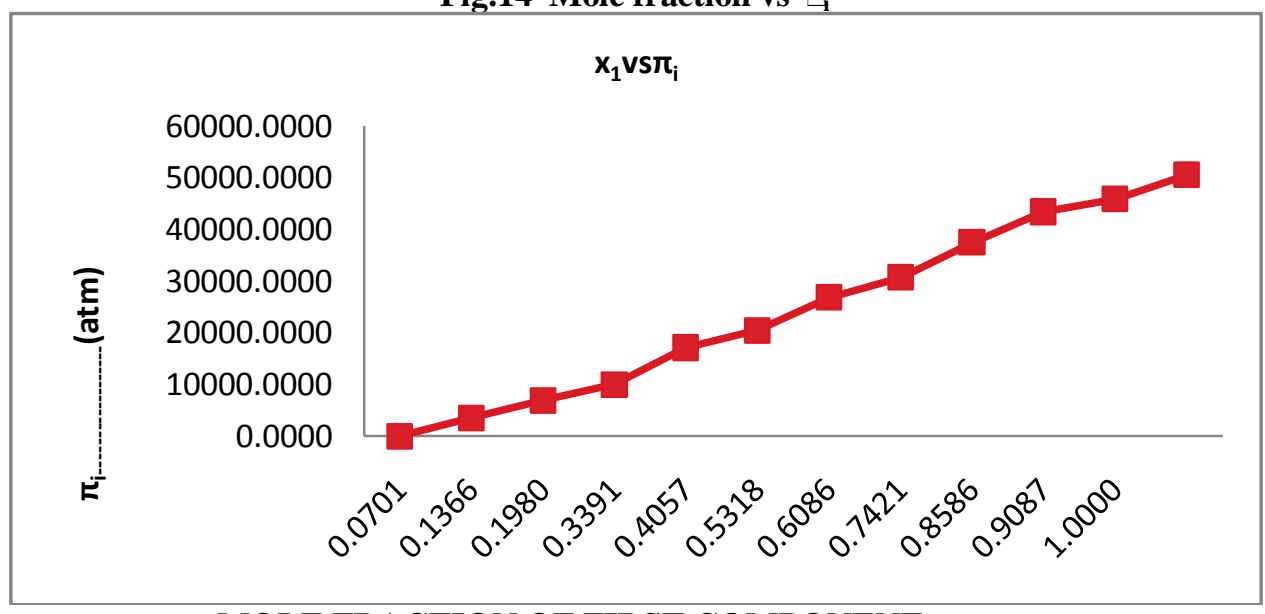

MOLE FRACTION OF FIRST COMPONENT

Fig.15 Mole fraction vs CE 


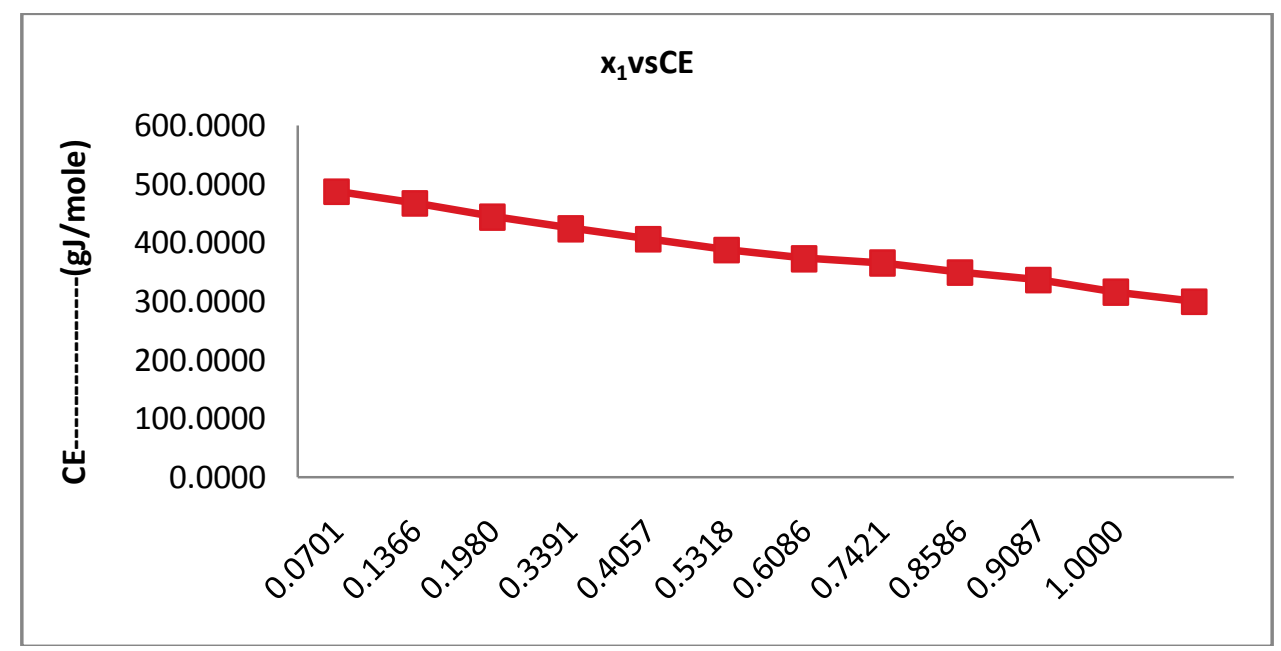

\section{MOLE FRACTION OF FIRST COMPONENT}

Fig.16 Mole fraction vs $\square$ G

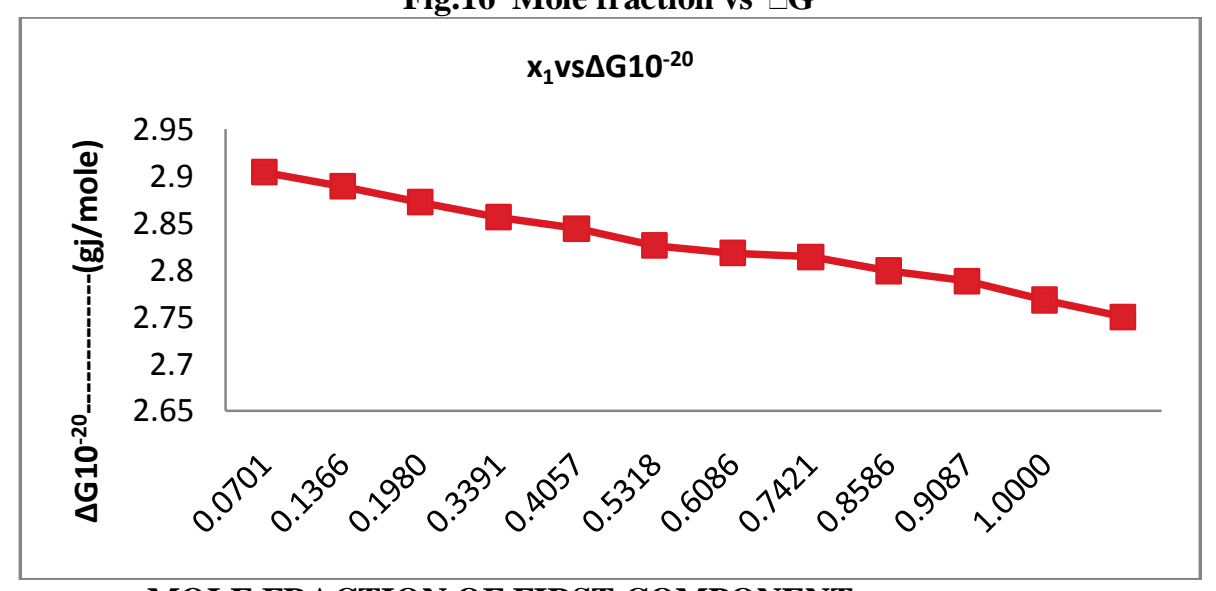

\section{MOLE FRACTION OF FIRST COMPONENT}

Table:-6 Excess ultrasonic velosity $\left(\mathrm{U}^{\mathrm{E}}\right)$,Excess viscocity $\left(\eta^{\mathrm{E}}\right)$, Excess available volume $\left(\mathrm{VA}^{\mathrm{E}}\right)$,Excess acoustical impedance $\left(\mathrm{Z}^{\mathrm{E}}\right)$, Excess volume $\left(\mathrm{V}^{\mathrm{E}}\right)$,Excess adiabatic compressibility $\left(\beta^{\mathrm{E}}\right)$,Excess free length $\left(\mathrm{L}_{\mathrm{F}}^{\mathrm{E}}\right)$ and Excess free volume $\left(\mathrm{V}_{\mathrm{F}}^{\mathrm{E}}\right)$ values at various mole fractions of IBMK+ N,N Dimethyl aniline at 308K.

\begin{tabular}{|c|c|c|c|c|c|c|c|}
\hline $\begin{array}{l}U^{\mathrm{E}} \\
(\mathrm{m} / \mathbf{s})\end{array}$ & $\left(\mathrm{NS} / \mathrm{cm}^{2}\right)$ & $\begin{array}{l}V^{\mathrm{E}} \\
\left(\mathrm{cm}^{\mathbf{B}}\right)\end{array}$ & $\begin{array}{l}Z^{\mathrm{E}} \\
\left(\mathrm{g}^{-2} \mathrm{~s}^{-1}\right)\end{array}$ & $\begin{array}{l}V^{\mathrm{E}} \\
\left(\mathrm{cm}^{3} / \mathrm{mole}\right)\end{array}$ & $\begin{array}{r}{ }^{\mathrm{E}} 10^{-9} \\
\left(\mathrm{~g}-{ }^{-1} \mathrm{~ms}^{2}\right)\end{array}$ & $\begin{array}{l}\mathbf{V}_{\mathbf{F}}^{\mathrm{E}} \\
\left(\mathbf{c m}^{3}\right)\end{array}$ & $\begin{array}{l}\mathbf{L}_{\mathbf{F}}{ }^{\mathrm{E}} \\
(\mathrm{cm})\end{array}$ \\
\hline 0.0 & $\mathbf{0 . 0 0 0 0}$ & $\mathbf{0 . 0 0 0 0}$ & 0.0000 & 0.0000 & 0.000 & $\mathbf{0 . 0 0 0 0}$ & $-\mathbf{- 0 . 0 5 0 0}$ \\
\hline 3.2 & -0.0650 & -0.2993 & 5.7942 & $-\mathbf{0 . 4 2 9 7}$ & -.104 & -0.0135 & 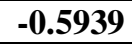 \\
\hline 3.8 & -0.1429 & -0.3343 & 5.1590 & $-\mathbf{- 0 . 4 5 0 0}$ & -.161 & -0.0178 & -0.8019 \\
\hline 6.4 & -0.1970 & -0.5376 & 6.7879 & 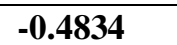 & -.227 & -0.0185 & -1.0726 \\
\hline 8.1 & -0.1728 & -0.6595 & 6.5589 & -0.5003 & -.318 & -0.0550 & -1.3981 \\
\hline 19.7 & -0.1933 & -1.5807 & $\mathbf{1 6 . 3 4 5 7}$ & -0.5119 & -.454 & -0.0498 & -2.0195 \\
\hline 1.5 & -0.1649 & -0.1379 & -0.2797 & -0.4961 & -.296 & -0.0728 & -1.2035 \\
\hline-7.0 & -0.1342 & $\begin{array}{c}.5304 \\
\end{array}$ & -7.5692 & -0.4769 & -.195 & $\begin{array}{c}-0.0894 \\
\end{array}$ & -0.7378 \\
\hline $\begin{array}{l}-5.7 \\
\end{array}$ & 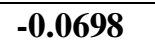 & $\begin{array}{c}-0.1832 \\
\end{array}$ & 17.1689 & $\mathbf{- 3 . 1 6 3 7}$ & -.328 & -0.1118 & -2.0575 \\
\hline-3.7 & -0.0132 & -0.3824 & $\mathbf{1 8 . 8 6 5 4}$ & -3.0659 & -.283 & -0.1204 & -1.8937 \\
\hline-11.3 & $-\mathbf{0 . 0 3 7 4}$ & $\mathbf{0 . 5 3 0 2}$ & 1.2706 & -1.5794 & -4.41 & -0.0519 & -0.5348 \\
\hline 0.0 & $\mathbf{0 . 0 0 0 0}$ & $\mathbf{0 . 0 0 0 0}$ & 0.0000 & 0.0000 & 0.000 & $\mathbf{0 . 0 0 0 0}$ & 0.0000 \\
\hline
\end{tabular}


Fig.17 Mole fraction vs $Z^{\mathrm{E}}$

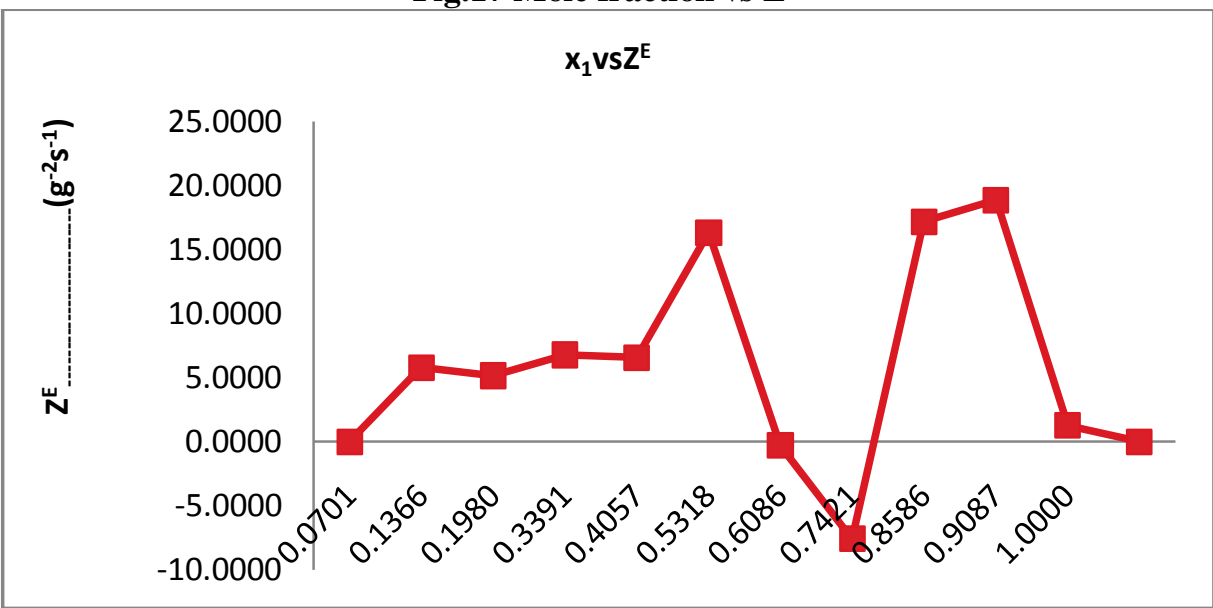

MOLE FRACTION OF FIRST COMPONENT

Fig.18 Mole fraction vs $V^{E}$

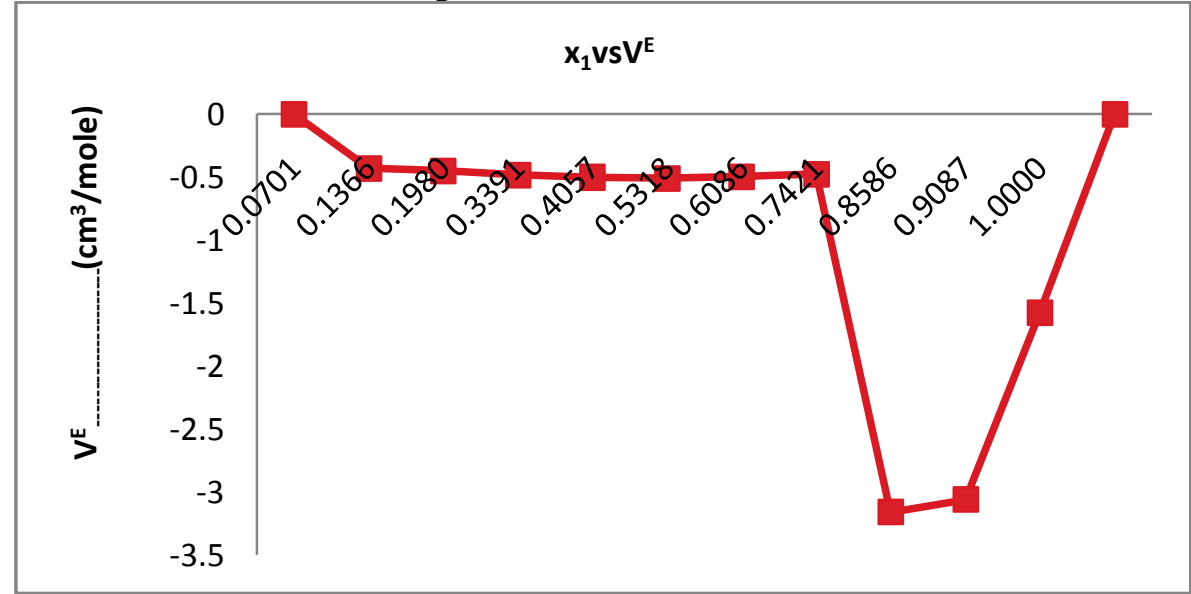

MOLE FRACTION OF FIRST COMPONENT

Fig.19 Mole fraction vs $V^{\mathrm{E}}$

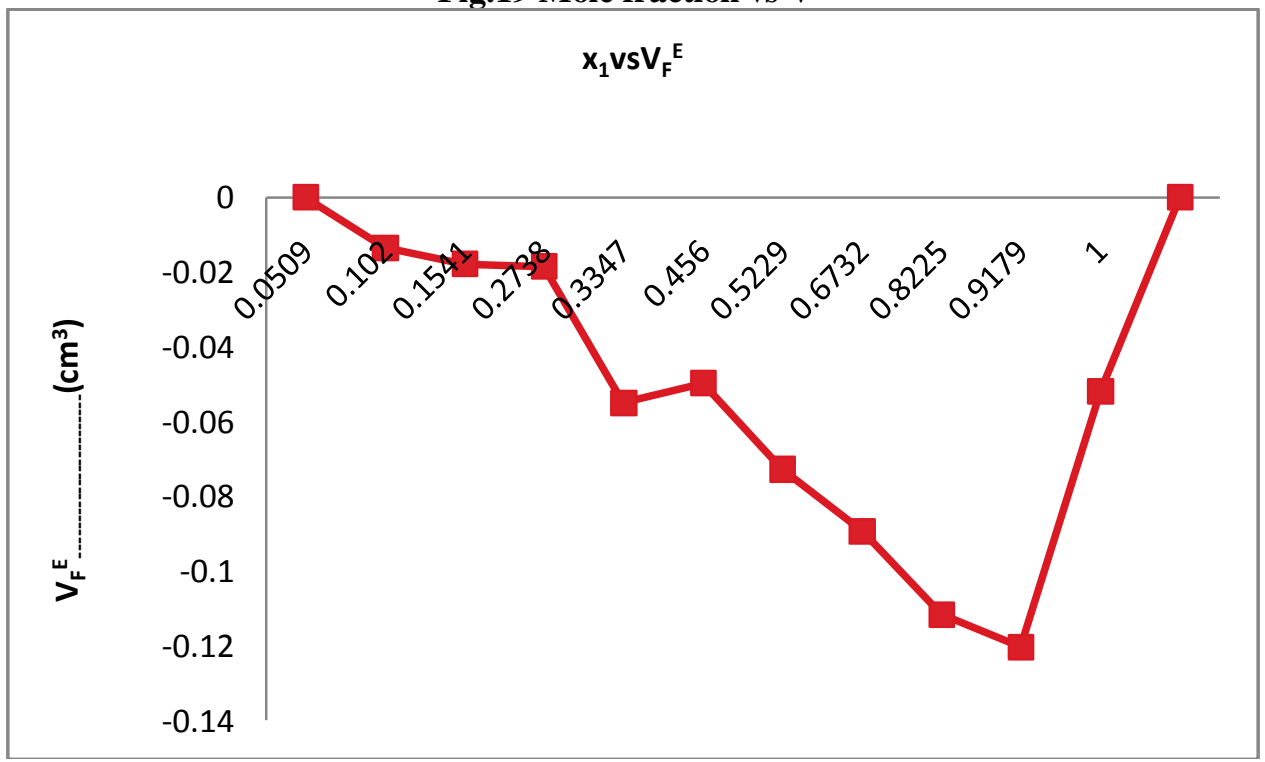

MOLE FRACTION OF FIRST COMPONENT 
Fig.20 Mole fraction vs $\mathbf{L}_{\mathrm{F}}{ }^{\mathrm{E}}$

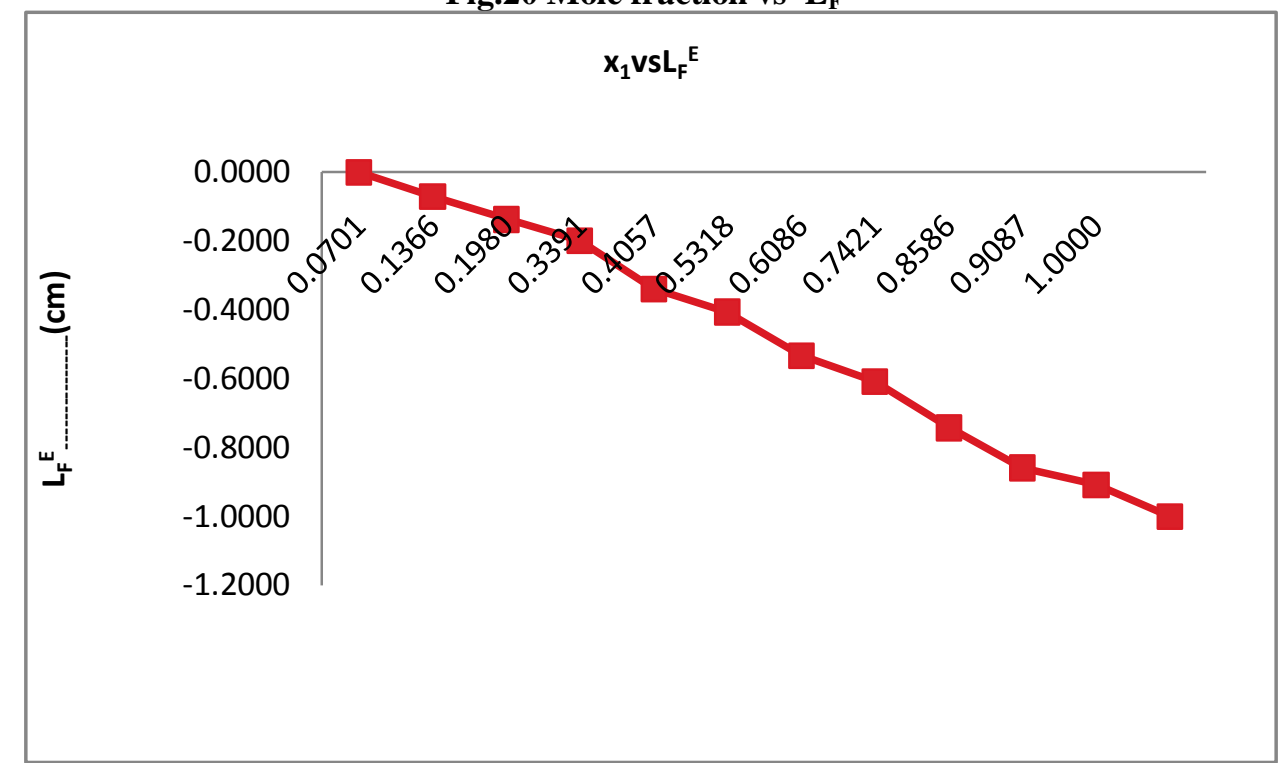

MOLE FRACTION OF FIRST COMPONENT

SYSTEM: 3 ACETOPHENONE+CYCLOHEXANE

Table:-7 Mole fraction of first component $\left(\mathrm{X}_{1}\right)$, Mole fraction of second component $\left(\mathrm{X}_{2}\right)$, Density $(\rho)$, $\operatorname{viscosity}(\eta)$, ultrasonic velocity(U), acoustic impedance(Z), Leonard's Jones potential(LJP) and Molecular interaction parameter $\left(\chi_{\mathrm{u}}\right)$ values at different mole fraction of Acetophenone+Cyclohexane at $308 \mathrm{~K}$.

\begin{tabular}{|c|c|c|c|c|c|c|c|}
\hline \multicolumn{2}{|c|}{ Mole fraction } & \multirow{2}{*}{$\begin{array}{l}\square \\
\text { (g/cm3) }\end{array}$} & \multirow{2}{*}{$\begin{array}{l}\square \\
\text { (cp) }\end{array}$} & \multirow{2}{*}{$\begin{array}{l}\mathbf{U} \\
(\mathbf{m} / \mathbf{s})\end{array}$} & \multirow{2}{*}{$\begin{array}{l}\mathrm{Z} \\
\left(\mathrm{gm}^{-2} \mathrm{~s}^{-1}\right)\end{array}$} & \multirow[b]{2}{*}{ LJP } & \multirow[b]{2}{*}{$\begin{array}{l}\square \mathrm{u} \\
(\mathbf{m} / \mathbf{s})\end{array}$} \\
\hline $\mathbf{X}_{1}$ & $\mathbf{X}_{2}$ & & & & & & \\
\hline 0.0000 & 1.0000 & 0.7517 & 0.5343 & 876.80 & 659.1337 & 13.2743 & 0.0000 \\
\hline 0.1019 & 0.8981 & 0.7583 & 0.5569 & 885.20 & 671.2397 & 13.4303 & -0.0712 \\
\hline 0.2013 & 0.7987 & 0.8042 & 0.5716 & 901.20 & 724.7447 & 13.7378 & -0.1228 \\
\hline 0.3036 & 0.6964 & 0.8289 & 0.6022 & 909.20 & 753.6110 & 13.8969 & -0.1764 \\
\hline 0.4019 & 0.5981 & 0.8576 & 0.6568 & 942.80 & 808.5363 & 14.6074 & -0.1993 \\
\hline 0.5011 & 0.4989 & 0.8663 & 0.7039 & 976.80 & 846.1888 & 15.4044 & -0.2196 \\
\hline 0.6067 & 0.3933 & 0.8870 & 0.8291 & 1228.80 & 1089.9654 & 1.3920 & -0.0766 \\
\hline 0.7020 & 0.2980 & 0.9113 & 0.8862 & 1389.20 & 1265.9566 & 45.5408 & -0.0092 \\
\hline 0.8055 & 0.1945 & 0.9548 & 0.9069 & 1476.00 & 1409.2343 & 77.4194 & -0.0024 \\
\hline 0.9044 & 0.0956 & 0.9770 & 1.0521 & 1528.00 & 1492.8743 & 133.3333 & -0.01640 \\
\hline 1.0000 & 0.0000 & 1.0023 & 1.1754 & 1625.20 & 1628.9246 & -380.9524 & 0.0001 \\
\hline
\end{tabular}

Fig.21 Mole fraction vs U

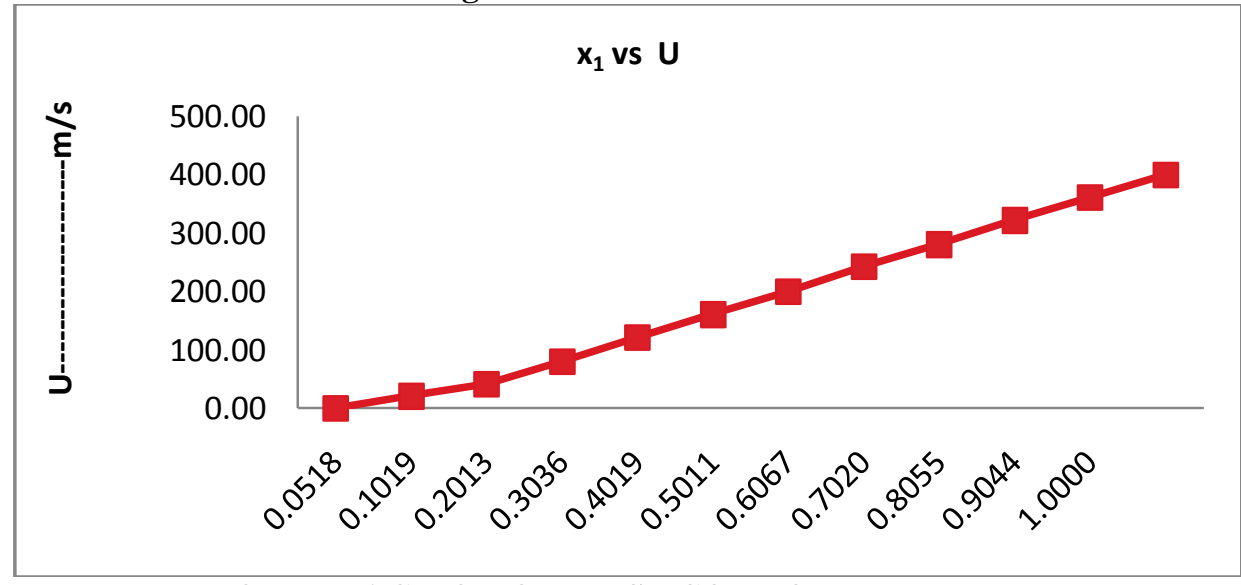

MOLE FRACTION OF FIRST COMPONENT 
Fig.22 Mole fraction vs $\square$

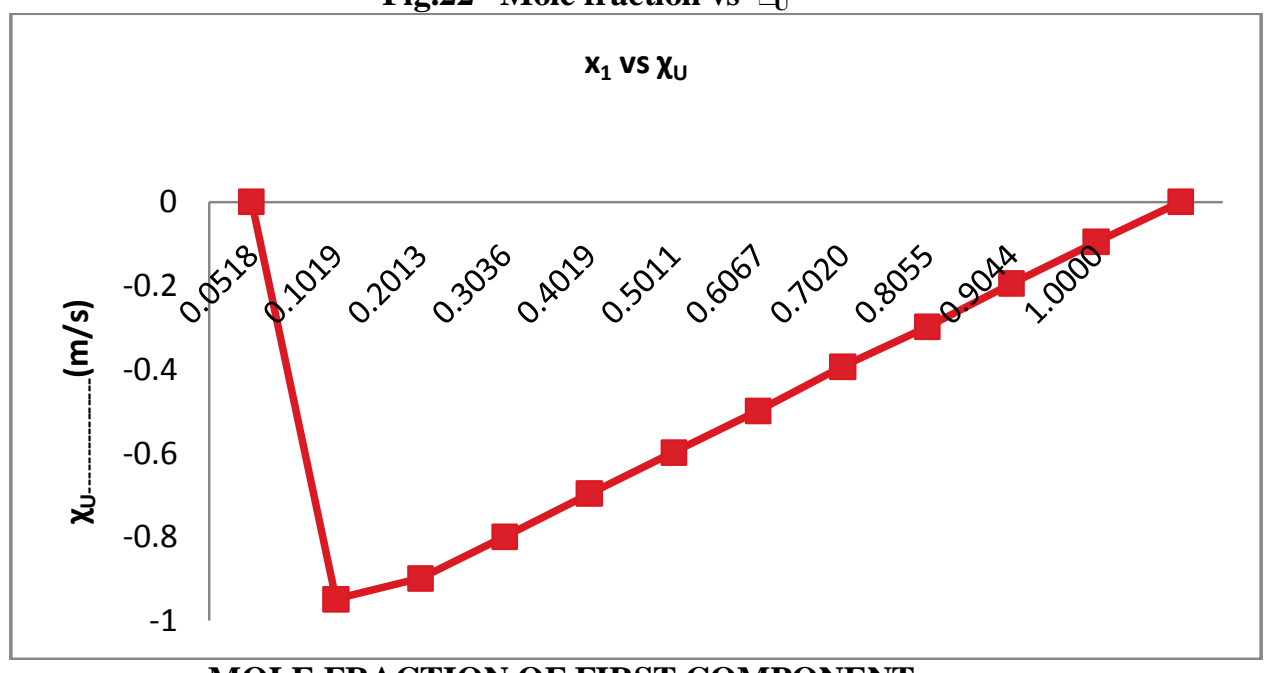

MOLE FRACTION OF FIRST COMPONENT

Table:-8 adiabatic compressibility $(\beta)$, relaxation time $(\tau)$, free volume $\left(\mathrm{V}_{\mathrm{f}}\right)$, internal pressure $\left(\pi_{\mathrm{i}}\right)$, cohessive force $(\mathrm{CE})$, absorption co-efficient $\left(\alpha / \mathrm{f}^{2}\right)$, free length $\left(\mathrm{L}_{\mathrm{f}}\right) \&$ activation energy $\left(\Delta \mathrm{G}^{\#}\right)$ values at different

\begin{tabular}{|c|c|c|c|c|c|c|c|}
\hline $\begin{array}{l}\square_{10}^{-12} \\
\left(\mathbf{T} . \mathbf{P a}^{)-1}\right.\end{array}$ & $\begin{array}{l}\square \mathbf{1 0}^{6} \\
\text { (s) }\end{array}$ & $\begin{array}{l}V_{f} \\
(\mathrm{ml} / \text { mole })\end{array}$ & $\begin{array}{l}\square_{\mathbf{i}} \\
(\mathbf{a t m})\end{array}$ & $\begin{array}{l}\text { CE } \\
\text { (gJ/mole) }\end{array}$ & $\begin{array}{c}\square / \mathbf{f}^{2} 10^{3} \\
\left(\mathrm{NPm}^{1} \mathbf{s}^{2}\right)\end{array}$ & $\begin{array}{l}\mathbf{L}_{\mathbf{f}} \\
\left(\mathbf{T} . \mathbf{P a}^{)-1}\right.\end{array}$ & $\begin{array}{l}\square \mathrm{G}^{\#} \mathbf{1 0}^{-20} \\
(\mathrm{Gj} / \mathrm{mole})\end{array}$ \\
\hline 173.03 & 1.2327 & 0.1833 & 3832.7939 & 429.0899 & 0.0413 & 166.7082 & 2.9334 \\
\hline 170.19 & 1.2476 & 0.1829 & 3796.7959 & 431.6480 & 0.0408 & 165.7495 & 2.9356 \\
\hline $168 . .2$ & 1.2498 & 0.1863 & 3726.9205 & 431.6620 & 0.0392 & 165.1981 & 2.9360 \\
\hline 153.10 & 1.1668 & 0.1954 & 3714.3895 & 422.1750 & 0.0357 & 158.9832 & 2.9233 \\
\hline 145.94 & 1.1719 & 0.1942 & 3698.7308 & 424.3108 & 0.0353 & 155.9086 & 2.9241 \\
\hline 131.18 & 1.1488 & 0.1902 & 3718.3730 & 427.6193 & 0.0337 & 150.5199 & 2.9204 \\
\hline 120.98 & 1.1354 & 0.1907 & 3652.5246 & 430.8831 & 0.0310 & 147.1330 & 2.9182 \\
\hline 74.663 & 0.8254 & 0.2224 & 3440.9151 & 411.1766 & 0.0159 & 129.6395 & 2.8594 \\
\hline 56.86 & 0.6718 & $\mathbf{0 . 2 5 3 7}$ & 3282.1172 & 394.1091 & 0.0104 & 120.2913 & 2.8213 \\
\hline 48.07 & 0.5813 & 0.2822 & 3195.5616 & 378.7082 & 0.0080 & 114.0124 & 2.7946 \\
\hline 43.83 & 0.6150 & 0.2492 & 3313.2958 & 395.7911 & 0.0088 & 110.7725 & 2.8050 \\
\hline 37.77 & 0.5920 & 0.2418 & 3338.8852 & 400.2498 & 0.0081 & 106.0458 & 2.7980 \\
\hline
\end{tabular}

Fig.23 Mole fraction vs

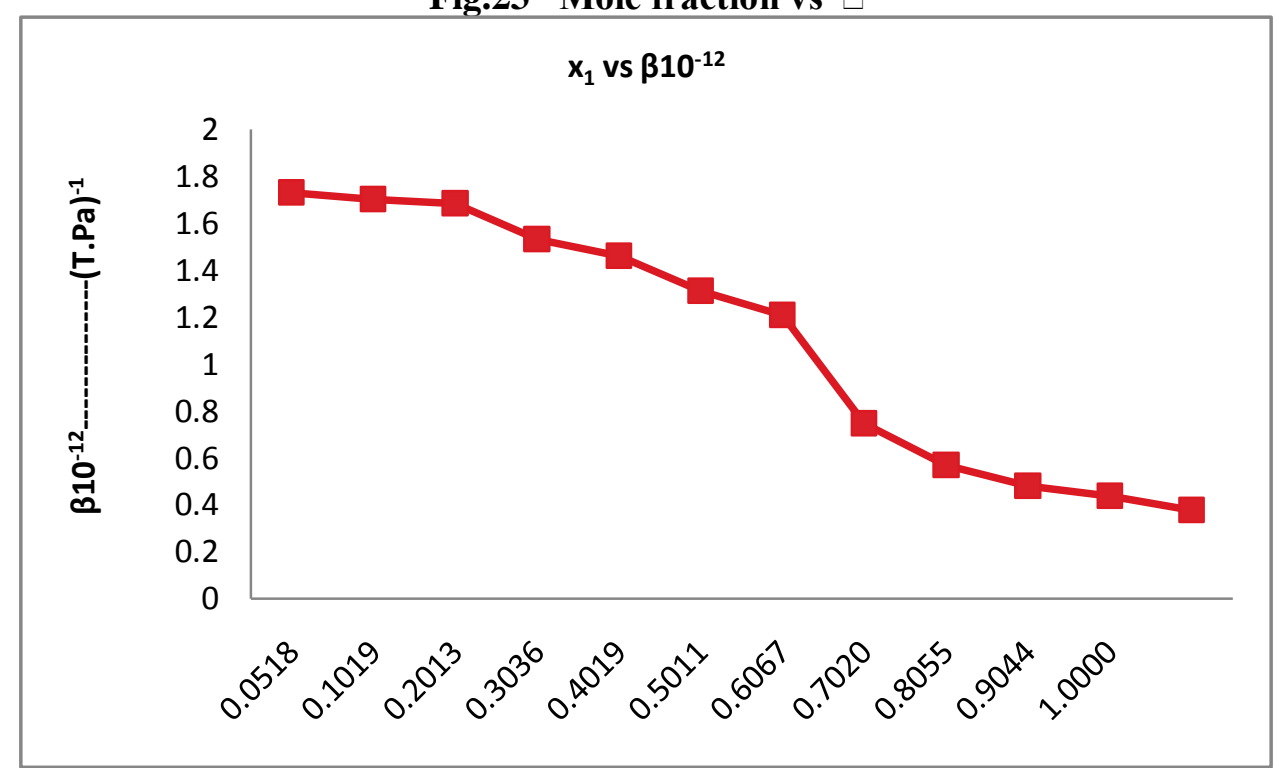

MOLE FRACTION OF FIRST COMPONENT 
Fig.24 Mole fraction vs $\square$

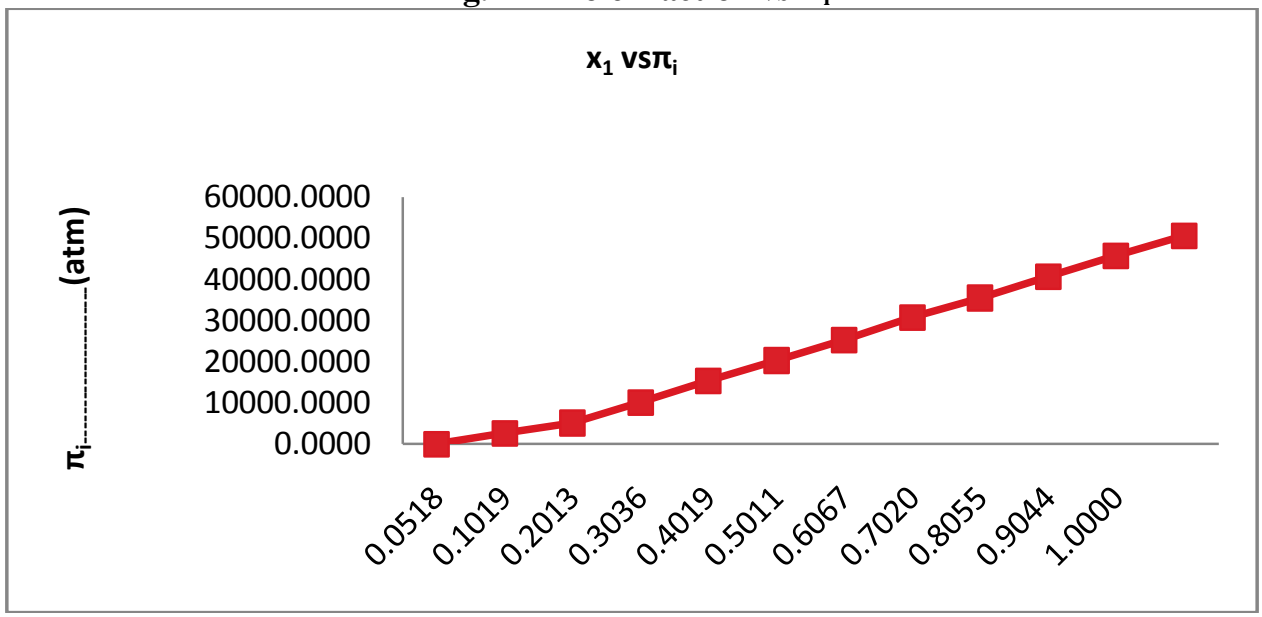

MOLE FRACTION OF FIRST COMPONENT

Fig.25 Mole fraction vs CE

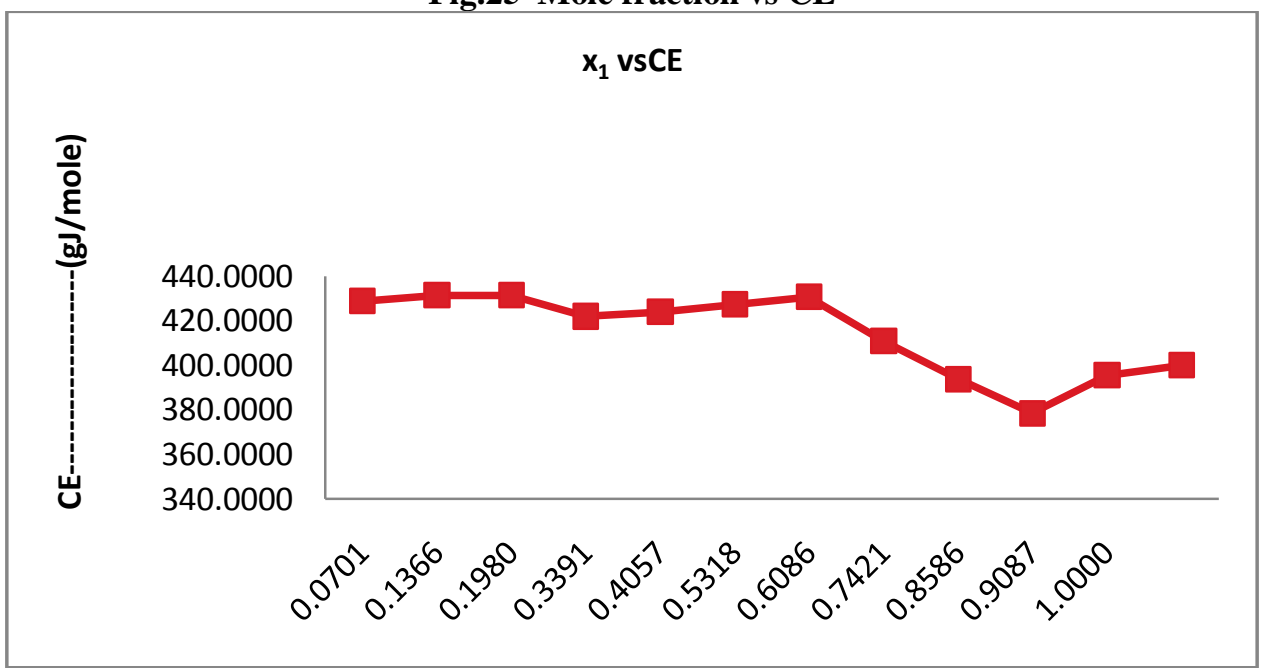

MOLE FRACTION OF FIRST COMPONENT

Fig.26 Mole fraction vs $\square \mathrm{G}$

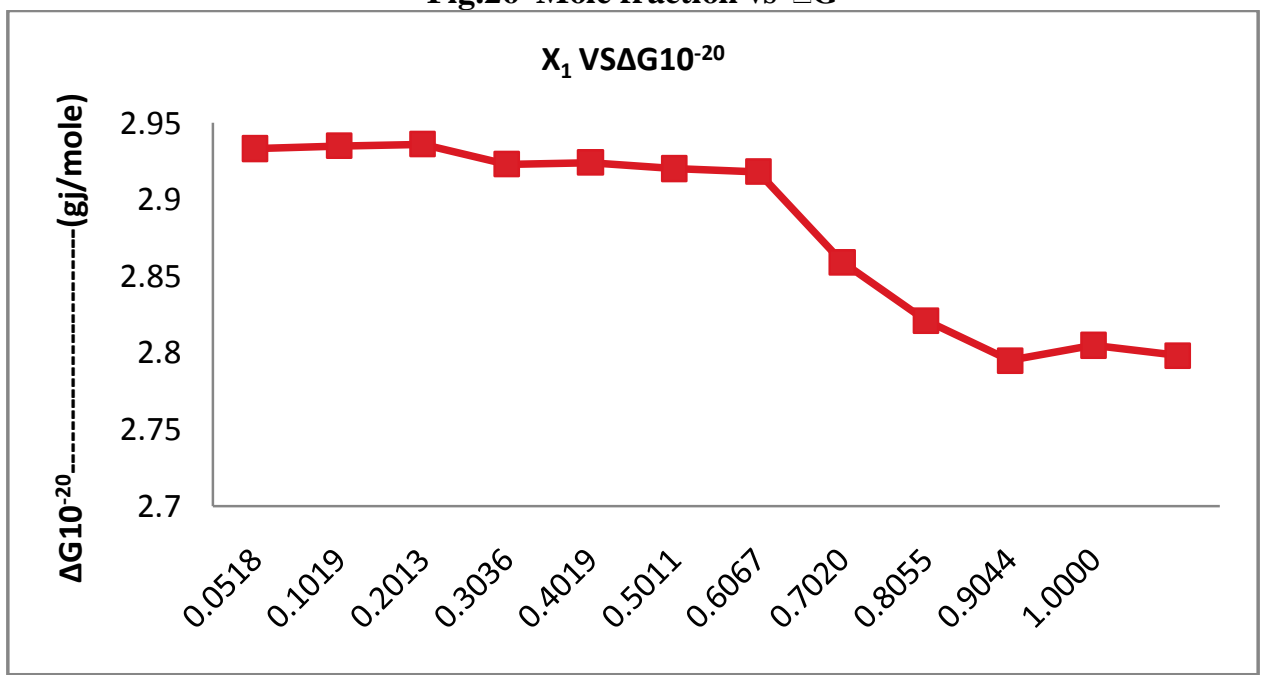

MOLE FRACTION OF FIRST COMPONENT 
Table:-9 Excess ultrasonic velocity $\left(\mathrm{U}^{\mathrm{E}}\right)$,Excess viscosity $\left(\eta^{\mathrm{E}}\right)$, Excess available volume $\left(\mathrm{VA}^{\mathrm{E}}\right)$, Excess acoustical impedance $\left(\mathrm{Z}^{\mathrm{E}}\right)$, Excess volume $\left(\mathrm{V}^{\mathrm{E}}\right)$,Excess adiabatic compressibility $\left(\beta^{\mathrm{E}}\right)$,Excess free length $\left(\mathrm{L}_{\mathrm{F}}^{\mathrm{E}}\right)$ and Excess free volume $\left(\mathrm{V}_{\mathrm{F}}^{\mathrm{E}}\right)$ values at various mole fractions of Acetophenone+Cyclohexane at $308 \mathrm{~K}$.

\begin{tabular}{|c|c|c|c|c|c|c|c|}
\hline $\begin{array}{l}\mathrm{U}^{\mathrm{E}} \\
(\mathbf{m} / \mathbf{s})\end{array}$ & $\begin{array}{l}\mathrm{E} \\
\left(\mathrm{NS} / \mathrm{cm}^{2}\right)\end{array}$ & $\begin{array}{l}\mathrm{VA}^{\mathrm{E}} \\
\left(\mathbf{c m}^{3}\right)\end{array}$ & $\begin{array}{l}Z^{E} \\
\left(g^{-2} s^{-1}\right)\end{array}$ & $\begin{array}{l}V^{\mathrm{E}} \\
\left(\mathrm{cm}^{3} / \mathrm{mole}\right)\end{array}$ & $\left(\mathrm{g}-{ }^{1} \mathrm{~ms}^{2}\right)$ & $\begin{array}{l}V_{\mathbf{F}}^{\mathrm{E}} \\
\left(\mathbf{c m}^{\mathbf{3}}\right)\end{array}$ & $\begin{array}{l}\mathbf{L F}^{\mathrm{E}} \\
(\mathbf{c m})\end{array}$ \\
\hline 0.0000 & 0.0000 & 0.0000 & 0.0000 & 0.0000 & \begin{tabular}{|l|l|}
-1.73 \\
\end{tabular} & 0.0000 & 0.0000 \\
\hline-34.4 & -0.0177 & 3.1907 & -42.5402 & 1.3179 & -1.64 & -0.0035 & 2.1836 \\
\hline-67.8 & -0.0427 & 6.4902 & -86.6215 & 3.0566 & -1.55 & -0.0030 & 4.6714 \\
\hline-126.2 & -0.0918 & 9.6046 & -129.4218 & 0.1065 & -1.38 & 0.0003 & 4.4863 \\
\hline-194.8 & -0.1267 & 14.8632 & -199.6705 & 0.3555 & -1.20 & -0.0068 & 7.6175 \\
\hline-234.7 & -0.1352 & $\mathbf{1 7 . 7 3 0 5}$ & -239.9848 & -0.1388 & -1.03 & -0.0166 & 8.1920 \\
\hline-274.9 & -0.1517 & 21.6493 & -298.4438 & 2.0429 & -0.863 & -0.0219 & 10.8227 \\
\hline-101.9 & -0.0941 & 496.3138 & -156.9795 & 2.7349 & -0.681 & 0.0036 & -0.2648 \\
\hline-12.8 & -0.0982 & 2.0661 & -73.3212 & 2.5620 & -0.516 & 0.0294 & -3.8319 \\
\hline-3.5 & -0.1438 & 0.8632 & -30.3212 & 0.1758 & -0.337 & 0.0518 & -3.8322 \\
\hline-25.5 & -0.0620 & 2.2454 & -42.5020 & 0.3378 & -0.165 & 0.0130 & -1.0726 \\
\hline 0.0000 & 0.0000 & 0.0000 & 0.0000 & 0.0000 & 0.0000 & 0.0000 & 0.0000 \\
\hline
\end{tabular}

Fig.27 Mole fraction vs $\mathrm{V}^{\mathrm{E}}$

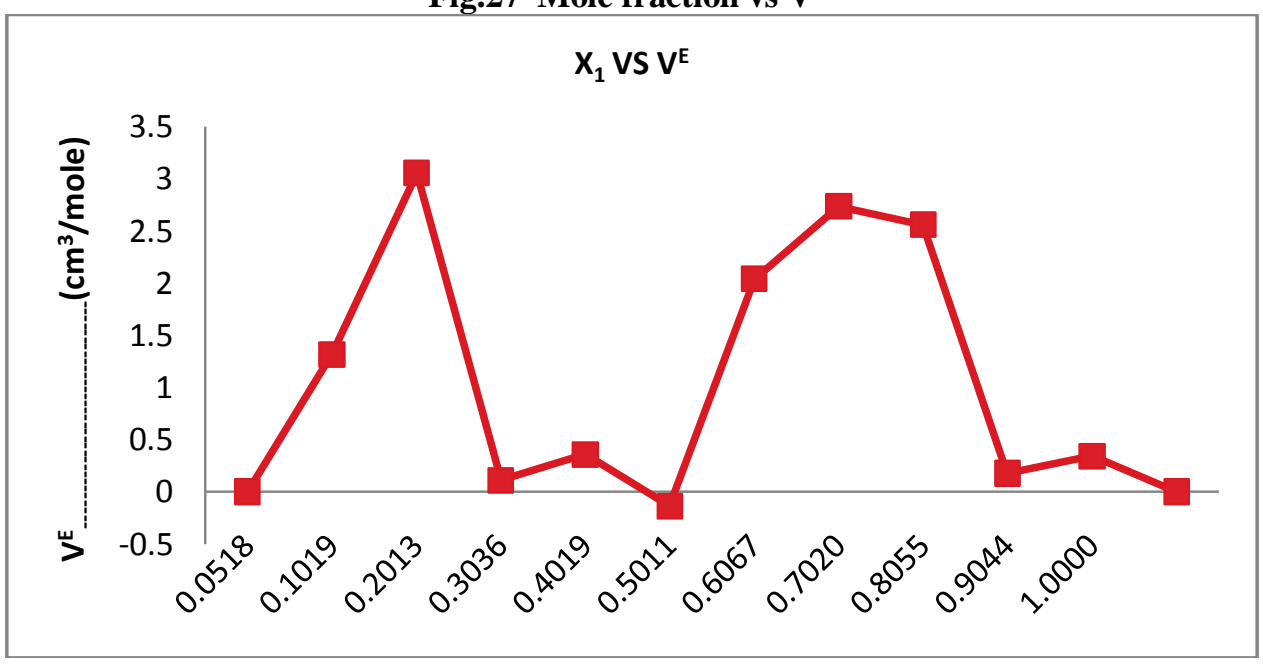

MOLE FRACTION OF FIRST COMPONENT

Fig.28 Mole fraction vs $\mathrm{Z}^{\mathrm{E}}$

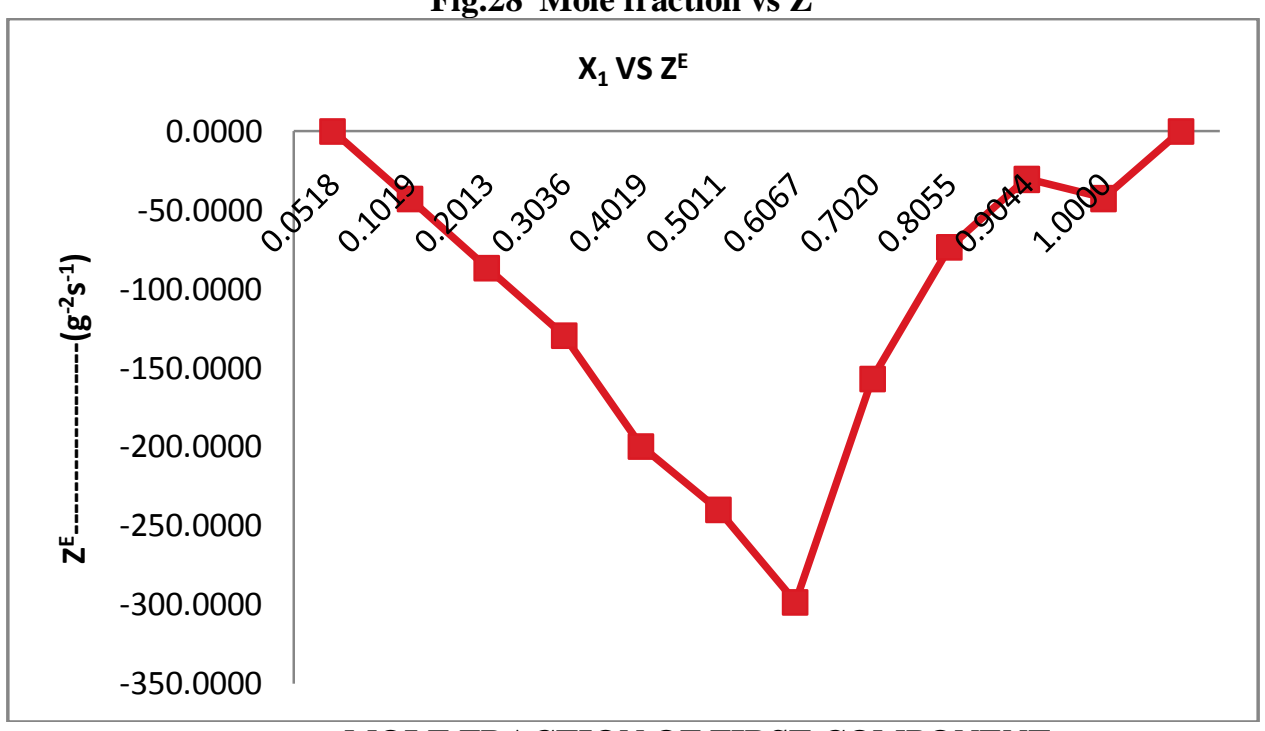

MOLE FRACTION OF FIRST COMPONENT 
Fig.29 Mole fraction $v_{S} V_{F}$

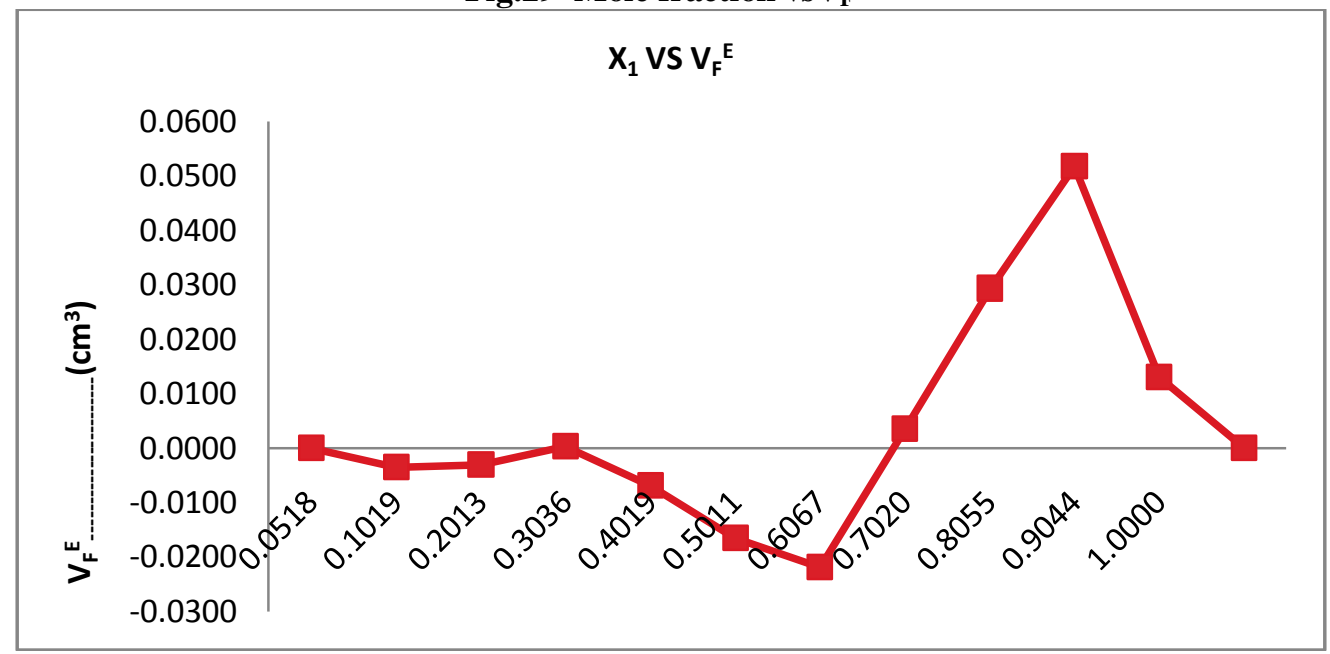

MOLE FRACTION OF FIRST COMPONENT

Fig.30 Mole fraction vsL ${ }_{F}{ }^{E}$

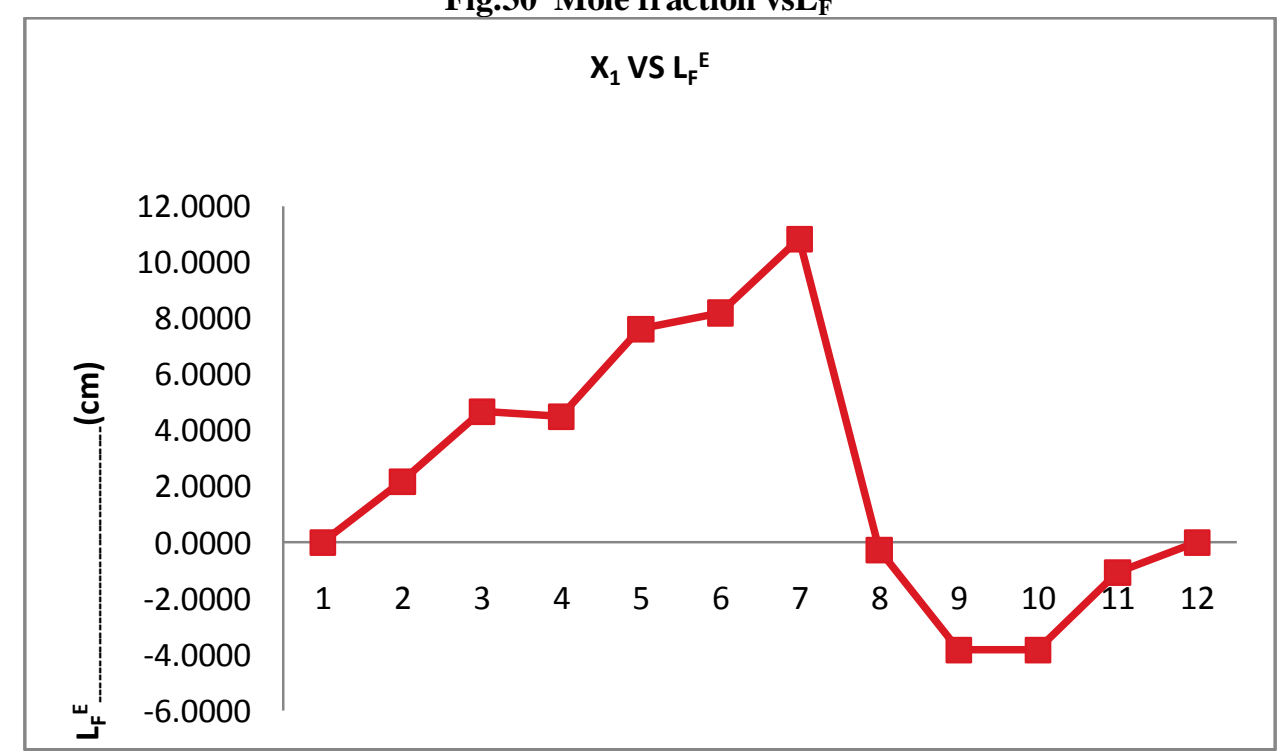

MOLE FRACTION OF FIRST COMPONENT

From the tables $1,4 \& 7$, The corresponding plots are given in Figs- 1,11,21. it is noted that the density decreases with increase in mole fraction For IBMK+anilinel,IBMK+N,N Dimethyl Aniline except Acetophenone+Cyclohexane. Ultrasonic velocity and viscosity decreases with increase in mole fraction of the solute in For IBMK+anilinel,IBMK+N,N -Dimethyl Aniline except Acetophenone+Cyclohexane system.

From the tables 2,5\&8, The corresponding plots are given in Figs- 2,12,22. it is noted that the decrease in velocity is due to the increase in free length and adiabatic compressibility. The decrease in velocity is due to the increase in free length and adiabatic compressibility of the liquid mixtures IBMK+anilinel,IBMK+N,N Dimethyl Aniline except Acetophenone+Cyclohexane. It is observed that for a given concentration as the number of - $\mathrm{CH}$ group or chain length increases, the sound velocity increases.

The adiabatic compressibility and free length increases with increase of mole fraction in IBMK+aniline,IBMK+N,N Dimethyl Aniline except Acetophenone+Cyclohexane systems. This may lead to the presence of specific molecular interaction between the molecules of the liquid mixture. The adiabatic compressibility and free length are the deciding factors of the ultrasonic velocity in liquid systems. The internal pressure decrease and free volume increases with increasing mole fraction.

From the tables 2,5\&8. The corresponding plots are given in Figs- 4,14,24. it is noted that the internal pressure may give information regarding the nature and strength of forces existing between the molecules. The decrease in free volume shows that the strength of interaction decreases gradually with the increase in solute concentration. It represents that there is weak interaction between the solute and solvent molecules like Acetophenone+Cyclohexane systems. 
When two liquids are mixed,there is a molecular attraction between the molecules of components and hence the cohesive energy is high.The cohesive energy and absorption coefficient values are decreased with increases in mole fractions in all the systems which may be due to weak induced dipole-induced dipole interactions in all systems..

From the tables 2,5,8. Acoustic impedance decreases with increase of mole fraction in IBMK+anilinel,IBMK+N,N - Dimethyl Aniline except Acetophenone+Cyclohexane systems. The relaxation time $(\tau)$ decreases with increasing concentration for all the three systems. The dispersion of the ultrasonic velocity in the system should contain information about the characteristic time $(\tau)$ )o the relaxation process that causes dispersion.

The relaxation time which is in the order of $10^{-12} \mathrm{sec}$ is due to structural relaxation process ${ }^{\mathbf{2 0}}$ and in such a situation it is suggested that the molecules get rearranged due to co-operative process ${ }^{21}$.

The Gibb's Free energy decreases with increasing mole fraction of all the systems.

From the table1,3\&7. The corresponding plots are given in Figs- 3,13,23. It is seen that the molecular interaction parameters values are more negative in system 2- IBMK+N,N Dimethyl Aniline \& system 3 Acetophenone+Cyclohexane than system 1- IBMK+anilinel. It is suggested that dipole-dipole interactions stronger than induced dipole-induced dipole interactions.

From the table2,5\&8. The corresponding plots are given in Figs- 6,16,26.The Gibb's Free energy decreases with increasing mole fraction of all the systems.This may be due to the intermediate compound formation between binary liquids. It is observed Generally free energy decrease favors the formation of products from reaction. This observation confirms the formation of hydrogen bonding in binary mixtures.

From the table3,6\&9. The corresponding plots are given in Figs- 7,8,9,10\&17,18,19,20 \&27,28,29,30.

The excess acoustical parameters can be used to find out the extent of deviation from ideal behavior in binary liquid mixtures. These values are calculated for all the three binary systems for different mole fractions at 308K. These values are presented in Tables 3, 6, 9. The corresponding plots are given in Figs-7, 8, 9, 10\&17, 18, 19,20 $\& 27,28,29,30$. It may be pointed out that the excess adiabatic compressibility $\left(\beta^{\mathrm{E}}\right)$, excess free length $\left(\mathrm{L}_{\mathrm{f}}^{\mathrm{E}}\right)$ and excess available volume $\left(\mathrm{V}_{\mathrm{a}}^{\mathrm{E}}\right)$ are negative for almost all compositions of system $2 \& 3$. This indicates that the attractive forces between the molecules of components are stronger than the intermolecular attractions in Acetophenone+Cyclohexane .

From the table 3,6\&9. Shows the values of excess adiabatic compressibility $\left(\beta^{\mathrm{E}}\right)$, excess free length $\left(\mathrm{L}_{\mathrm{f}}^{E}\right)$, excess free volume $\left(\mathrm{V}_{\mathrm{f}}^{E}\right)$ for IBMK+Aniline system than IBmk+N,N-Dimethylaniline,Acetophenone+ Cyclohexane system at $308 \mathrm{~K}$. From the Table 3,6,\&9, it is observed that as the concentration of IBMK increases the ultrasonic velocity decreases for both the systems studied.

As shown in Table3,6\&9. $\beta^{\mathbf{E}}$ values are negative which suggest the presence of hydrogen bonding interaction between the components of the liquid mixtures. However, $\beta^{\mathrm{E}}$ values are positive which suggest that absence of hydrogen bonding.

This indicates that the less interaction in the Acetophenone+Cyclohexane than IBMK+aniline, IBMK+N, N-Dimethyl aniline system. The possible reason may be as follows, in the Acetophenone+cyclohexane, the closeness of $-\mathrm{CO}-\mathrm{CH}_{3}$ to $-\mathrm{C} \mathrm{H}$ group shows the presence of two types of effect. One is the increase of electron density in the $-\mathrm{CO}-\mathrm{CH}_{3}$ bond and the other is the resonance effect.

These two effects decrease the strength of the intermolecular hydrogen bond formation in acetophenone+cyclohexane system than IBMK+ aniline, IBMK+ N, Ndimethyl aniline system. Hence from these factors, there is less intermolecular hydrogen bond formation and less dipole-dipole interaction in Acetophenone+Cyclohexane system.

\section{Conclusion}

The computed acoustical parameters and their values point to the presence of specific molecular interaction in the liquid mixtures IBMK+aniline, IBMK+N,Ndimethyl aniline system than acetophenone+cyclohexane system.

Hence it is concluded that the association in these mixtures is the result of strong Hydrogen bonding between the molecules \& strong Dipole-Dipole interactions Ibmk+ aniline,Ibmk+ N,Ndimethyl aniline system than Acetophenone+Cyclohexane system in Binary liquid mixtures.

IBMK+aniline> IBMK+N,Ndimethyl aniline> Acetophenone+Cyclohexane

\section{Acknowledgment}

The author thank to Prof. Dr. Neeraja, Department of chemistry, Adhiyamann college of engineering, Hosur-635 109, India. who helped and guided me during my research and also, my sincere thanks to Dr.Raghunath sir, principal of Adhiyamann college of engineering as well Honorable chairman Munivenkateppan, Sri venkateswara institute of engineering college, Krishnagiri. 


\section{References}

[1]. $\quad$ Nikam P S Jagdale B S Sawant A B and Mehdi Hasan, Indian J Pure Appl Phys.,2001, 39, 433.

[2]. Shipra Baluja and Swati Oza, Fluid Phase Equilib., 2001, 178, 233.

[3]. Ali A, Nain A K and Kamil M, Thermochim.Acta, 1996, 274, 209.

[4]. Manisha Gupta and Shukla J P, Indian J Pure Appl Phys., 1996, 34, 772

[5]. Pankaj and Sharma C, Ultrasonics, 1991, 29, 344.

[6]. Velmurugan s, Nambinarayanan T K, Srinivasa Rao A and Krishnan B, Indian J Phys., 1987, 61B, 105.

[7]. Suryanarayana C V, J Acoust Soc Ind., 1983, 13, 11.

[8]. Fletcher A, J Phys Chem., 1969, 73, 2217.

[9]. Pimental G C and Maclellan A L, The Hydrogen Bond, (WH Freeman and Co, San Fransisco) 1960.

[10]. Hobbs M E and Bates WW, J Am Chem Soc., 1952, 74, 746.

[11]. Lin W and Tsay S J, J Phys Chem., 1970, 74, 1037.

[12]. Kannappan A N, Kesavasamy R and Ponnuswamy V, ARPN Journal of Engineering and Applied. Sciences, $2008,3,41$.

[13]. Glasstone, S., Laidler, K.J., and Erying, H., Theory of Rate Processes.

[14]. Mc. Graw - Hill, Newyork, 478, 479, (1950). Erying, H.and Kincaid, J.F.J. Chem. Phy., 6,520,(1938).

[15]. Richards, T.W., Chem. Rev., 2,315, (1925).

[16]. Vanderwaals, J.d., Essay on the continuity of the gaseous and liquid States London (1873).

[17]. Glasstone, S., Thermodynamic for chemist, D. van Mostrand Co., Inc., Newyork, 62, (1947).

[18]. Ratha, D.C., Mishra, S.C. and Samal, K., J.Pure. Appl. Ultrason., 12,108, (1990).

[19]. Kinsler, L.E. and Rray. A.R., Fundamentals of Acoustics (Wiley Eastern, New Delhi). (1989).

[20]. Ali, A., Hyder, S. and Nain, A.K., Ind.J. Phys. 74 B, 63 (2000).

[21]. Sumathi T and Uma Maheswari, Indian J Pure \&Appl Phys, 2009, 47, 782.

\section{Author profile}

\section{Author}

The author D.Ubagaramary has been currently involved in doing her Ph.D in Acoustical Studies on Molecular Interactions in Binary Liquid Mixtures at $308 \mathrm{~K}$ through ultrasonic measurements under the guidance of Prof. Dr.P.Neeraja, Department of Chemistry, Adhiyamann College of engineering, Hosur-635 109, India.

She has completed her M.Phil in the field of Ultrasonics at Bharathdasan University, India, under the guidance of Dr.X, .Rosario rajkumar Department of Chemistry, St.Joseph's college in Trichy, India. This author is currently working as an Assistant Professor in Sri Venkateswara institute of engineering, Krishnagiri, India. Also, the author has been qualified in M.sc., M.phil. B.Ed., 